\title{
Porpagation of femtosecond laser pulses in litharge Index Sf57: Time-frequency
}

\author{
Mounir Khelladi \\ Faculty of Technology, Telecommunication Department, University of Tlemcen, Tlemcen, Algeria
}

\section{Email address:}

khelladi.photonic@yahoo.fr

\section{To cite this article:}

Mounir Khelladi. Porpagation of Femtosecond Laser Pulses in Litharge Index Sf57: Time-Frequency. American Journal of Physics and Applications. Special Issue: Laser Applications in Physics and Biophotonics. Vol. 3, No. 1-1, 2015, pp. 1-17. doi: 10.11648/j.ajpa.s.2015030101.11

\begin{abstract}
An efficient numerical algorithm is presented for the numerical modeling of the propagation of ultrashort pulses with arbitrary temporal and frequency characteristics through linear homogeneous dielectrics. The consequences of proper sampling of the spectral phase in pulse propagation and its influence on the efficiency of computation are discussed in detail. The numerical simulation presented here is capable of analyzing the pulse in the temporal-frequency domain. As an example, pulse propagation effects such as temporal and spectral shifts, pulse broadening effects, asymmetry and chirping in dispersive media are demonstrated for wavelet decomposition.
\end{abstract}

Keywords: Ultrashort Laser Pulse, Index Refraction, Femtosecond, Propagation, Time-Frequency Decomposition, Characterizations, Frog, Spider, Vampire

\section{Introduction}

Recent developments in short pulse laser technology have led to significant advances in our fundamental understanding of ultrafast phenomena in physics, chemistry and biology, as well as stimulating the development of applications in fields as diverse as optical communications, biomedical imaging and femtosecond micromachining (Florenta,2006).

Ultrafast laser technology has advanced over recent years to the point that pulses with high peak power and a duration of less than $10 \mathrm{fs}$ can be generated directly from mode-locked Ti:sapphire lasers. Propagation of ultrashort optical pulses in a linear optical medium consisting of free space (Chritov, 1985) (Ziolkowski, 1992), (Sheppard, 1997), (Agrawal, 1998), (Kpalan, 1998), dispersive media (Porras, 1999), diffractive optical elements (Ichikawa, 1999),(Piestun, et. Al 2001), focusing elements (Matei, 1999) and apertures (Liu, 2004), (Hwang, 2003) has been extensively studied analytically; though only a few isolated attempts have been made on numerical simulation. However, analytical methods have the limitations of not being able to handle arbitrary pulse profiles.

Propagation of ultrashort optical pulses in a linear optical medium consisting of free space (Chiritov 1985, Agrawal 1998), dispersive media (Kaplan 1998, Agrawal 1999), diffractive optical elements (Porras 1999, Chikawa 1999), focusing elements (Piestun 2001, Kempe 1999) and apertures has been extensively studied analytically, though only a few isolated attempts have been made on numerical simulation. Most studies are based analytical calculations assuming a plane wave or $\mathrm{TEM}_{00}$ Gaussian transverse profile and a Gaussian temporal profile for the pulse. The change in the spectral properties of the pulse on propagation of the pulse was investigated analytically by Sheppard and Gan (Sheppard, 1999) taking special forms of Gaussian pulsed beams. Agrawal considered spatial broadening of diffracted pulses assuming Gaussian transverse and temporal profile. However, analytical methods have the limitations of not being able to handle arbitrary pulse profiles. Also, closed form solutions are often obtained after certain levels of approximations. This has motive a few studies based on the use of numerical simulation techniques in the analysis of pulse propagation. For example, Kaplan introduced numerical evaluation by fast Fourier transform to analyze pulses of arbitrary temporal profile and investigated on-axis temporal evolution of the pulse in the far field. In view of the recent advance in ultrashort pulse propagation, a strong need is felt for developing a numerical formalism capable of performing such a complete analysis of the issues involved in pulse propagation. 
Here we introduce a numerical simulation tool for propagation of ultrashort pulses of arbitrary shape through linear homogeneous media based on wave optical field representation which enables an easy evaluation for the merit functions of the pulsed field. This allows us to analyze the pulse in the time-frequency domain at any arbitrary plane. With this tool, we investigate the spectral and temporal evolution of ultrashort pulses at any arbitrary propagation distance. The propagation of the pulse is achieved in terms of its spectral equivalent. Further, we introduce certain sampling rules for the spectral phase so that the phase information is sampled properly when we move from one spectral component to another in the spectral equivalent of the pulse. As a consequence, the algorithm becomes computationally efficient since we only considered a small number of spectral components for simulation of pulse propagation.

\section{Ultarshort Laser Pulses Generations}

The central aim of this section is to give a concise introduction to nonlinear optics and to provide basic information about the most-widely used tunable femtosecond laser sources, in particular tunable Ti:sapphire oscillators and Ti:sapphire amplifiers or optical parametric amplifiers.

\subsection{The Ti:Sapphire Oscillator}

In 1982, the first Ti:sapphire laser was built by (Moulton,1982). The laser tunes from $680 \mathrm{~nm}$ to $1130 \mathrm{~nm}$, which is the widest tuning range of any laser of its class 1 . Nowadays Ti:sapphire lasers usually deliver several watts of average output power and produce pulses as short as $6.5 \mathrm{fs}$.

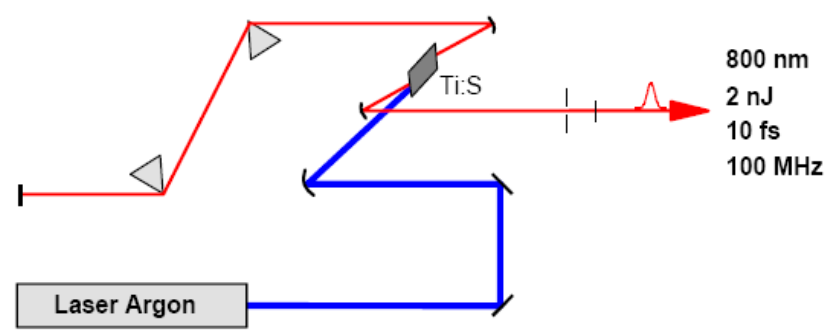

Fig 1. A Ti: sapphire oscillator. The compensation of dispersion speed of group is ensured by both prisms, producing a negative dispersion speed of group. The blocking of mode is ensured by Kerr effect. On the one hand, in the temporal field, the non-linear phase makes it possible to compensate for the negative quadratic component resulting from the dispersion of the cavity in linear mode, which makes it possible to have perfectly equidistant longitudinal modes. In addition, in the space field, self focusing by a Kerr lens makes it possible to decrease the losses when the various longitudinal modes are in phase, thus privileging the pulse mode.

\subsubsection{The Kerr Lens Mode-Locking}

For simplicity, consider the spatial propagation of a Gaussian laser beam in a nonlinear material. The intensity profile of the beam is a function of its radius $r$ and of a shape parameter $g$ [12]. At high intensities, the refractive index depends nonlinearly on the propagating field. The lowest order of this dependence can be written as follows:

$$
n(r)=n_{0} \frac{1}{2} n_{2} I(r)
$$

where $n_{2}$ is the nonlinear index coefficient and describes the strength of the coupling between the electric field and the refractive index $n$. The intensity is:

$$
I(r)=e^{-g r^{2}}
$$

Hence, the refractive index changes with intensity along the optical path and it is larger in the center than at the side of the nonlinear crystal. This leads to the beam self-focusing phenomenon, which is known as the Kerr lens effect (see Fig.2). This process is enhanced along the optical path

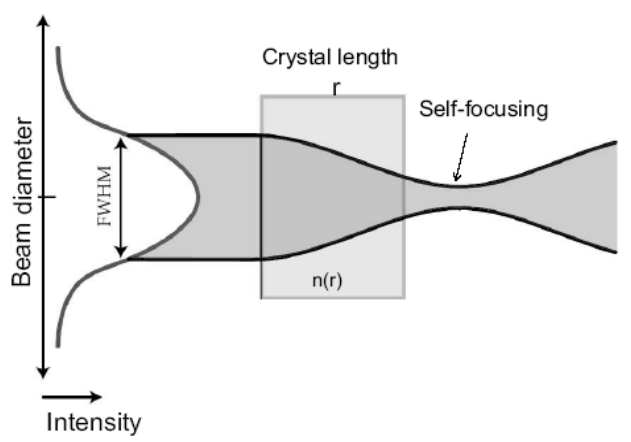

Fig 2. The Kerr lens effect and self-focusing. The index of refraction varies with intensity along the beam diameter. Depending on the sign of the nonlinear term $n_{2}$ in the expression (1) the index of refraction increases or decreases towards the center of the laser beam. For positive $n_{2}$ the laser beam self-focuses.

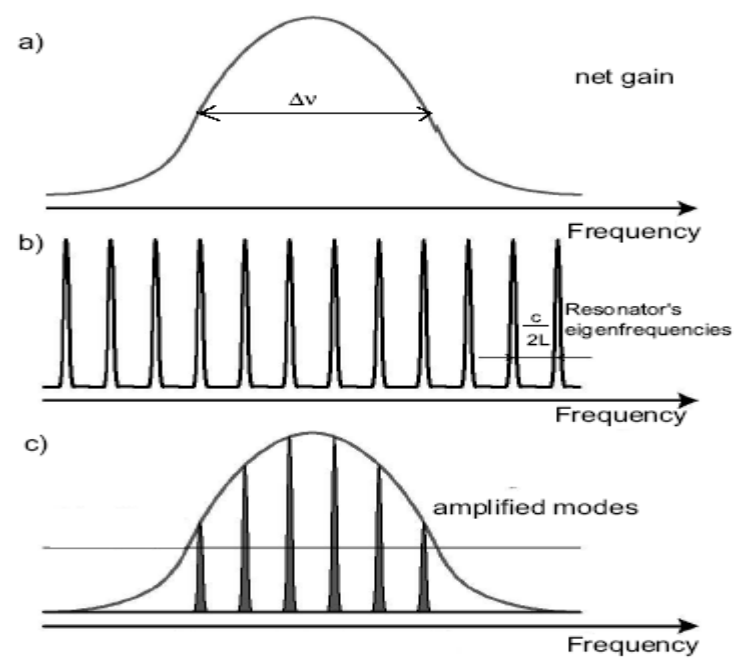

Fig 3. The Kerr Lens Mode-Locking (KLM) principle. The axial modes of a laser cavity are separated by the intermode frequency spacing $v=c / 2 L$. (a) The net gain curve (gain minus losses). In this example, from all the longitudinal modes in the resonator (b), only six (c) are forced to have an equal phase.

because focusing the beam increases the focal "power" of this "lens". The increase of the focusing stops when the diameter of the beam is small enough and the linear diffraction is large enough to balance the Kerr effect. Consider now a seed beam with a Gaussian profile propagating through a nonlinear medium, e.g. a Ti:sapphire crystal, which is pumped by a cw radiation. As aforementioned, for high intensity light, due to 
the intensity dependent refractive index, the Kerr lens effect occurs. When the laser operates in its most usual regime (free-running laser), it can oscillate simultaneously over all the resonance frequencies of the cavity. These frequencies make up the set of longitudinal modes of the laser. For the stronger focused frequencies, the Kerr lens favors a higher amplification. Thus the self-focusing of the seed beam can be used to suppress the cw operation, because the losses of the $\mathrm{cw}$ radiation are higher. Forcing all the modes to have equal phase (mode-locking) implies that all the waves of different frequencies will interfere (add) constructively at one point, resulting in a very intense, short light pulse. The pulsed operation is then favored and it is said that the laser is modelocked. Thus the mode-locking occurs due to the Kerr lens effect induced in the nonlinear medium by the beam itself and the phenomenon is known as Kerr-lens mode-locking.

The modes are separated in frequency by $v=c / 2 L, \mathrm{~L}$ being the resonator length, which also gives the repetition rate of the mode-locked lasers:

$$
\tau_{\text {rep }}=\frac{1}{T}=\frac{c}{2 L}
$$

Moreover the ratio of the resonator length to the pulse duration is a measure of the number of modes oscillating in phase. For example if $L=1 \mathrm{~m}$ and the emerging pulses have $100 \mathrm{fs}$ time duration, there are 105 modes contributing to the pulse bandwidth. There are two ways of mode-locking a femtosecond laser: passive mode-locking and active modelocking.

Briefly, active mode-locking implies that the radiation in the laser cavity is modulated by a signal coming from an external clock source (e.g. acousto-optical modulator (AOM), electro-optical modulator). The modulation frequency of the AOM is continuously adjusted to match the reciprocal cavity round trip time $\tau_{\text {rep }}$ by an algorithm which records the instantaneous repetition rate of the laser. This method, also

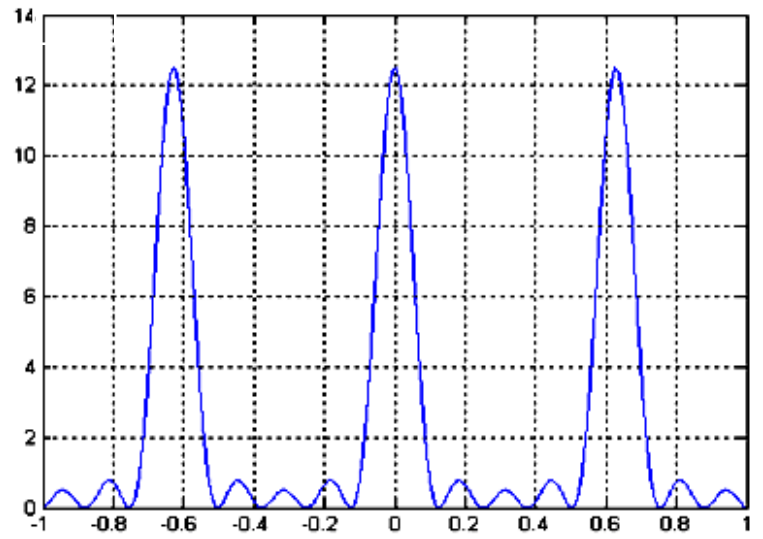

known as regenerative mode-locking, is used to initialize mode-locking in the Ti:sapphire oscillator (Spectra Physics Tsunami), where the AOM works at $80 \mathrm{MHz}$.

The passive mode-locking technique does not require an external clock in the laser resonator. Here the laser radiation itself generates a modulation through the action of a nonlinear device (e.g. moving a prism into or out of the cavity) situated in the resonator. The modulation is automatically synchronized to the cavity round trip frequency. The Ti:sapphire oscillator (Kapteyn-Murnane) uses the passive mode-locking technique.

Modelocking, as the word suggests, involves locking a large number of longitudinal modes of a laser in phase. In a modelocked laser, the electric fields associated with the different modes add constructively at one point and destructively elsewhere to create a short spike number of frequency components with same phase gives an infinite series of short pulses in time (Rulliere, 1998). In a laser cavity, these modes are equally spaced (with a spacing depending on the cavity length). The electric field distribution with $\mathrm{N}$ such modes in phase (considered to be zero, for convenience) can be written as:

$$
E(t)=\sum_{n}^{N-1} E_{n} e^{i w_{0}+n \Delta w t} \propto \frac{e^{i N \Delta w t}-e^{i w_{0} t}}{e^{i \Delta w t}-1}
$$

Where $w_{0}$ is the central frequency and $\Delta \mathrm{w}$ is the mode spacing, this appears as a carried wave with frequency domain. The laser intensity is given by

$$
I(t) \propto[A(t)]^{2}=\frac{\sin ^{2}[(2 n+1) \Delta w \cdot t / 2]}{\sin ^{2}(\Delta w \cdot t / 2)}
$$

This is series of pulses with width inversely proportional to the number of modes that are locked in phase of the mode spacing. The concept of mode-locking is easier said than done.

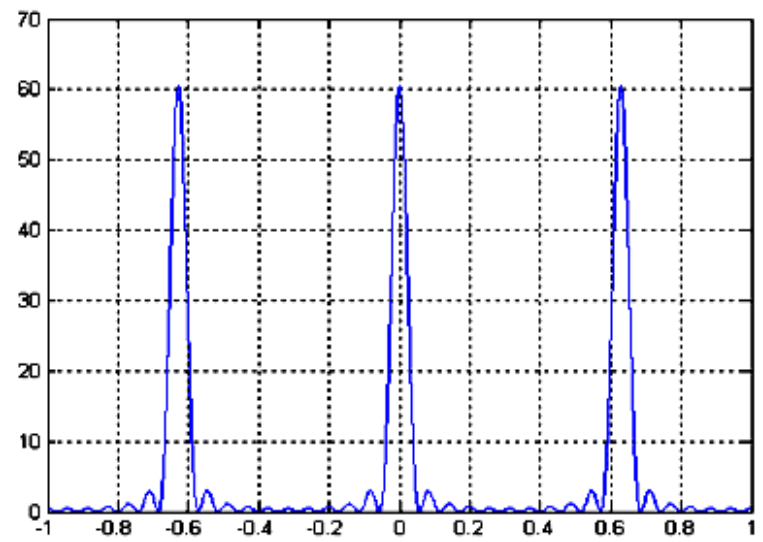

Fig 4. The influence of the phase relation between oscillating modes on the output intensity of the oscillation. (a) two modes in phase, and (b) five modes in phase.

Fig.4. shows how the time distribution of a laser output depends upon the phase relations between the modes. Fig.4.a is the resultant intensity of two modes in phase Fig.4.b, is the resultant intensity of five modes in phase and a period repetition of a wave packet from the resultant constructive interference can be seen. In a general continuous wave multimode laser, the modes will oscillate independently from each other and will have random phases relative to one another. 
One of the many advantages of Ti:sapphire oscillator is its high repetition rate $(70-100 \mathrm{MHz})$. This allows a good duty cycle where the clusters in the molecular beam are irradiated several times by the output laser pulses of a relatively low energy (several $\mathrm{nJ}$ ). Thus the laser operates in the weak-field regime. This avoids perturbation of potential energy surfaces (PES) produced by the intense laser pulse, simplifying the interpretation of the results and theoretical description. Moreover, non-resonant two-photon processes are not expected to occur.

\subsubsection{CPA Laser System}

Chirped pulse amplification (CPA) is a technique to produce ultrashort strong pulses. Although a CPA output can be very strong, it is poorly tunable in a small range around its output central wavelength. Various optical techniques have been innovated to make the output pulses tunable and/or shorter while keeping the pulse power as high as possible. CPA is the abbreviation of chirped pulse amplification. Chirped pulse amplification is a technique to produce a strong and at the same time ultrashort pulse. The concept behind CPA is a scheme to increase the energy of an ultrashort pulse while avoiding very high peak power in the amplification process.

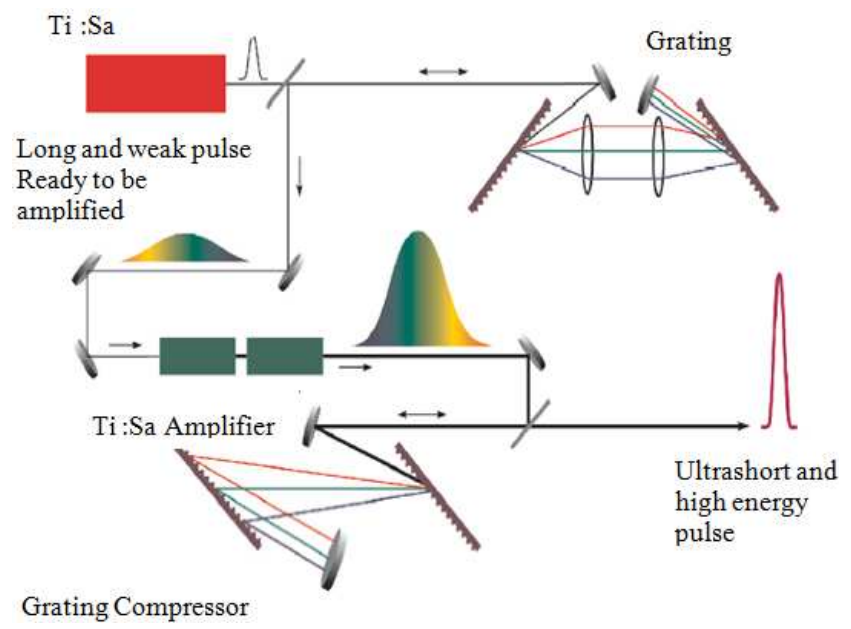

Fig 5. Schematics of a chirped pulse amplification system, showing the duration and energy level of the signal at the different stages of the system.

In the CPA technique, ultrashort pulses are generated typically at low energy $10^{-9} \mathrm{~J}$, with a duration around $10^{-12}-$ $10^{-14}$ seconds and at a high repetition rate of about $10^{8} 1 / \mathrm{s}$ in an oscillator. To amplify these ultrashort pulses without any damage to the amplifier, they first are stretched. The stretched pulses now have a duration of order the of a few hundred picoseconds. These chirped pulses can then be amplified in one or two amplification stages (Ruiz, et al, 2005).

In a CPA system, there are basically three components: a stretcher, an amplifier and a compressor; the first and latter components are basically the same for all CPA systems. For the amplifier stage there are two commonly used designs: multi-pass and regenerative (Joanna, 2007).

\subsection{Multipass and Regenerative Amplification}

Two of the most widely used techniques for amplification of femtosecond laser pulses are the multipass and the regenerative amplification. In the multipass amplification different passes are geometrically separated (see Fig.6.a). The number of passes (four to eight) are usually limited by the difficulties on focussing all the passes on a single spot of the crystal. The amplifier bandwidth has to be broad enough to support the pulse spectrum. This is why Ti:sapphire crystals are widely used in the amplification process.

The regenerative amplification technique implies trapping of the pulse to be amplified in a laser cavity (see Fig 2.b). Here the number of passes is not important. The pulse is kept in the resonator until all the energy stored in the amplification crystal is extracted. Trapping and dumping the pulse in and out of the resonator is done by using a Pockel cell (or pulsepicker) and a broad-band polarizer. The Pockel cell consists of a birefringent crystal that can change the polarization of a travelling laser field by applying high voltage on it. In the regenerative amplifier the Pockel cell is initially working like a quarter-wave plate. When the pulse is sent to the resonator, the voltage on the Pockel cell is switched (delay one) and it becomes the equivalent of a half-wave plate. In this way the pulse is kept in the cavity until it reaches saturation. Then a second voltage is applied (delay two) and the pulse is extracted from the resonator (Holzwarth, 2000).
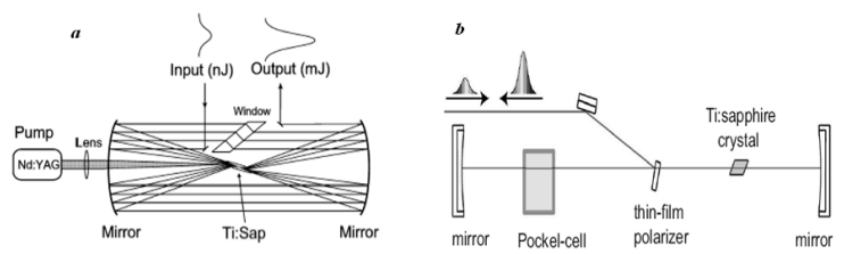

Fig 6. (a) The amplifier configuration uses two spherical mirrors in a multipass confocal Configuration to make the signal pass eight times through the amplifying medium, (b) Schematic principle of a regenerative amplifier.

Of all potential amplifier media, titanium- doped sapphire has been the most wide spread used. It has several desirable characteristics which make it ideal as a high power amplifier medium such as a very high damage threshold $\left(\sim 8-10 \mathrm{~J} / \mathrm{cm}^{2}\right)$, a high saturation fluence, and high thermal conductivity.

\subsection{Stretcher-Compressor}
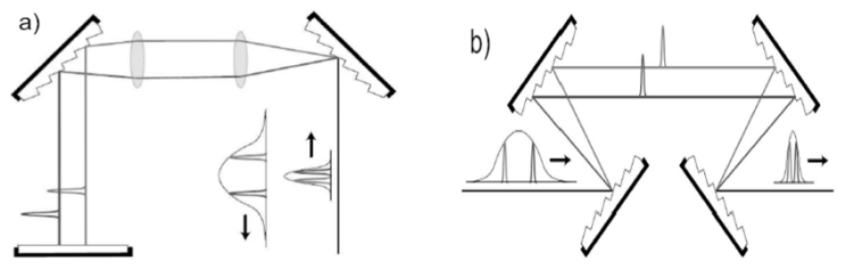

Fig 7. Principle of a stretcher (a) and a compressor (b). The stretcher setup extends the temporal duration of the femtosecond pulse, whereas the gratings' arrangement in the compressor will compress the time duration of the pulse. Both setups are used in femtosecond amplifiers.

By using a dispersive line (combination of gratings and/or lenses), the individual frequencies within a femtosecond 
pulse can be separated (stretched) from each other in time (see Figure 7a).

\subsection{Compressors}

In normal materials, low frequency components travel faster than high frequency components; in other words, the velocities of large wavelength components are higher than that of shorter ones. These materials induce a positive group velocity dispersion on a propagating pulse. To compensate the positive GVD and rephase the dephased components a setup which produces negative group velocity dispersion is needed. Some designs have been devised to produce negative group velocity dispersion, like grating compressor, prism compressor, Gires-Tournois interferometer and etc. They are called compressors, because they compress a pulse (in time domain) which is broadened due to dispersion and chirp. The grating compressor and the prism compressor are two conventional setups (Holzwarth, 2000).

\subsubsection{Grating Compressor}

Four identical gratings in a sequence as shown in Fig.8 make up a grating compressor. A pulse impinges on the first gratings with an angle of $\theta$. Different wave length components of the pulse spectrum are diffracted off with different angles from the first grating. Orientation of the grating is such that the longer wavelength path to the second grating is longer than the shorter wavelengths pathes. Then from the second grating the spectral components in the spectrum travel together in parallel directions but with wavelength dependent position (spatially chirped). The gratings are set in such a way that their wavelength dispersions are reversed which implies that the exiting ray from the second grating is parallel to the incident ray to the first grating.

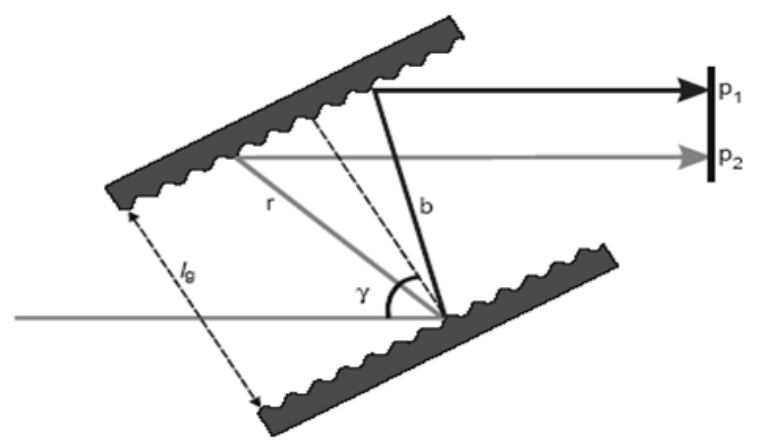

Fig 8. Grating pairs used in the control of dispersion. $r$ and $b$ indicate the relative paths of arbitrary long- and short-wavelength rays. $\gamma$ is the (Brewster) angle of incidence at the prism face. Light is reflected in the plane (p1-p2) in order to remove the spatial dispersion shown.

Travelling through the third and the forth gratings recombines all the components and removes the spatial chirp. It is possible to compact the design more by inserting a mirror in the plane of symmetry of the two gratings pairs. The beam will be reflected back and pass through the second and then the first grating. To compensate a certain amount of group velocity dispersion or third order dispersion of a pulse incoming to the compressor we need to know how much GVD and TOD are produced by the compressor (Holzwarth, 2000). The group delay induced by the grating compressor is given

$$
\mathrm{T}_{\mathrm{g}}=\frac{2 \mathrm{~L}}{\mathrm{c} \cdot \sqrt{1-\left(\frac{\lambda}{\mathrm{d}}-\sin \gamma\right)^{2}}}\left[1+\left(\frac{\lambda}{\mathrm{d}}-\sin \gamma\right) \sin \gamma\right]
$$

where, is the light wavelength, $\mathrm{L}$ is the distance between the gratings, $\mathrm{d}$ is the grating's constant and $\gamma$ is the incidence angle of the beam to the first grating. Dispersion of the group delay is obtained as:

$$
\mathrm{GDD}=\frac{\mathrm{d}^{2} \emptyset}{\mathrm{dw}^{2}}=-\frac{\lambda^{3} \mathrm{~L}}{\pi \mathrm{c}^{2} \mathrm{~d}^{2}}\left[1-\left(\frac{\lambda}{\mathrm{d}}-\sin \gamma\right)^{2}\right]^{-3 / 2}
$$

The third order dispersion produced in the grating compressor will be:

$$
\mathrm{TOD}=\frac{1}{\mathrm{~L}} \frac{\mathrm{d}^{3} \emptyset}{\mathrm{dw^{3 }}}=-\frac{\mathrm{d}^{2} \emptyset}{\mathrm{dw^{2 }}} \frac{6 \pi \lambda}{\mathrm{c}}\left[\frac{1+\frac{\lambda}{\mathrm{d}} \sin \gamma-\sin ^{2} \gamma}{1-\left(\frac{\lambda}{\mathrm{d}}-\sin \gamma\right)^{2}}\right]
$$

\subsubsection{Prism Compressor}

A prism compressor is built of four sequentially arranged identical prisms used in a geometry similar to Fig.9 (left); often at their minimum deviation (to decrease geometrical (spatial) distortion of prisms on the beam) and in their Brewster angle (to minimize power loss). Because of the symmetry in the arrangement, it is possible to place a mirror after the second prism (as we did in the grating compressor setup) perpendicular to the beam propagation direction. The first prism spreads the pulse spectral components out in space. In the second prism the red frequencies of the spectrum must pass through a longer length in the glass than the blue frequencies.

By moving the prism in and out of the beam path, the amount of the prism glass that the beam passes through can be changed for exact balancing of the pulse dispersion. The back reflected beam will pass through the prisms at a height slightly higher or lower than the incoming beam. To calculate the group velocity dispersion and the third order dispersion produced by the prism compressor we assume that the beam inside the compressor propagates near apices of the prisms.

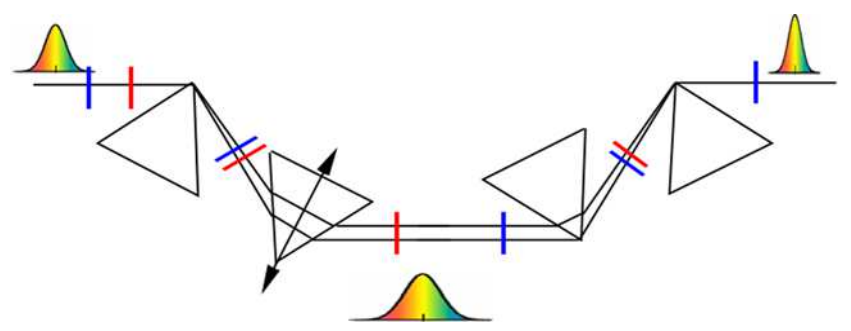

Fig 9. Pulse compressor, with a negative GDD. Longer wavelengths traverse more glass. The use of two prisms and a mirror simplifies the device somewhat, but it remains difficult to vary its GDD over a wide range and to tune it.

The optical path of a ray propagating in the compressor is defined as (Holzwarth, 2000): GDD will result 


$$
G D D=\frac{4 L \lambda^{3}}{2 \pi^{2} c^{2}}\left\{\sin \beta\left[\frac{d^{2} n}{d \lambda^{2}}+\left(\frac{d n}{d \lambda}\right)^{2}\left(2 n-\frac{1}{n^{3}}\right)\right]-2 \cos \beta\left(\frac{d n}{d \lambda}\right)^{2}\right\}
$$

The third order dispersion can also be evaluated in the same manner to that used above

$$
T O D=-\frac{L^{4}}{4 \pi^{2} c^{3} L}\left[3 \frac{d^{2} p}{d \lambda^{2}}-\lambda \frac{d^{3} p}{d \lambda^{3}}\right]
$$

\subsection{Mathematical Description of Laser Pulses}

In order to understand the behavior of ultrashort light pulses in the temporal and spectral domain, it is necessary to formulate the relation between the two domains mathematically.

They are linked through a Fourier transform, and so the modification of a laser pulse in one of the two domains either by propagation due to dispersion or actively by pulse shaping implies a modification of its properties in the conjugate domain as well. It is important to introduce the concept of the amplitude and the phase of the electric field because the generation, measurement, and shaping of ultrashort laser pulses is based on measuring and influencing these properties. The time behavior of a laser pulse at a fixed point in space including the envelope, the oscillations and the phase can be fully described by the real electric field $E(t)$. The electric field in the time-domain is invariably connected with its counterpart $E(w)$ in the frequency-domain via a Fourier transform:

\subsubsection{Pulse Duration and Spectral Width}

The statistical definitions are usually used in theoretic calculations and given as

$$
\left\langle t^{2}\right\rangle=\frac{\int_{-\infty}^{+\infty} t^{2}|E(t)|^{2} d t}{\int_{-\infty}^{+\infty}|E(t)|^{2} d t} ;\left\langle w^{2}\right\rangle=\frac{\int_{-\infty}^{+\infty} w^{2}|E(w)|^{2} d w}{\int_{-\infty}^{+\infty}|E(w)|^{2} d w}
$$

where $t$ is the intensity weighted average time. Duration of very complex pulse cannot be defined in the direct and simple way and statistical definitions is only approximation. In case of Gaussian pulse is easy to determine pulse duration and spectral width by applying FWHM of intensity. One can show that these quantities are related through the following universal inequality

$$
\Delta w \Delta \tau \geq 1 / 2
$$

For instance, a Gaussian-shaped pulse with pulse duration of $100 \mathrm{fs}$ has a minimum spectral bandwidth of $4.41 \mathrm{THz}$. Relation (12) can only be fulfilled for the case when $\Delta w \Delta \tau$ is directly equal $1 / 2$ with Gaussian temporal and spectral envelopes and the pulse is said to be a Fourier-transform limited or unchirped pulse. In this case, the instantaneous frequency is a time-independent quantity.

In practice, half-maximum quantities are easier to measure. Therefore, one defines the pulse duration $\Delta \tau$ as the Full Width at Half Maximum (FWHM) of the intensity profile and the spectral width $\Delta w$ as the FWHM of the spectral intensity. The Fourier inequality is then usually given by

$$
\Delta w \Delta \tau \geq K
$$

where $\mathrm{K}$ is a numerical constant, depending on the assumed shape of the pulse.

\section{Gaussian pulse}

In this subsection we would like to consider an example of Gaussian pulse, which is most commonly used in ultrashort laser pulse characteristics. The pulse is linearly chirped an represented by

$$
A(t)=A_{0} \exp \left(\frac{-(1+i \alpha) t^{2}}{\tau_{g}^{2}}\right) \text { with } \Delta \tau_{p}=\sqrt{2 \ln 2 \tau_{g}}
$$

The instantaneous frequency is given as

$$
w(t)=w_{0}+\frac{d \varphi(t)}{d t}=w_{0}-\frac{2 \alpha}{\tau_{g}^{2}} t
$$

Pulse is down-chirped for a positive chirp parameter $\alpha$, for negative is upchirped and when $\alpha=0$ then the pulse is unchirped. The spectral instensity can be derived by taking the Fourier-transform of eq.14, it also has the Gaussian shape (RULLIERE,1998)

If follows directly from eq. 13 that the minimum achievable duration is limited by the spectrum of the pulse. In other words, in order to produce ultrashort pulses a very broad spectral bandwidth is needed. The shortest possible pulse, for a given spectrum, is known as the transform-limited pulse duration. It should be noted that eq.13 is not equality, i.e. the product can very well exceed $K$. If the product exceeds $K$ the pulse is no longer transform-limited and all frequency components that constitute the pulse do not coincide in time, i.e. the pulse exhibits frequency modulation is very often referred to as a chirp.

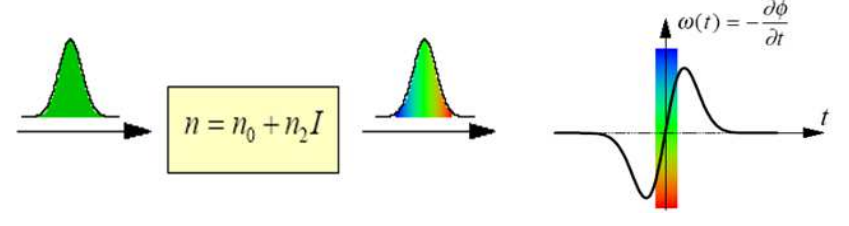

Fig 10. Self-phase modulation. Variation in the instantaneous frequency $w(t)$ of the transmitted pulse after the propagation through a nonlinear medium with a positive nonlinear index of refraction, $n 2$.

Ultrashort laser pulses are coherent bursts of electromagnetic radiation, confined in time and space. They are characterized by several parameters: temporal coherence, spatial coherence (i.e. focusing ability) contrast, power, etc. Here the description is concentrated on their temporal aspects.

\subsubsection{Time Domain Description}

Since in this paper the main emphasis is on the temporal dependence, all spatial dependence is neglected, i.e., $E(x, y, z, t)=E(t)$. the electric field $E(t)$, is a real quantity and all measured quantities are real. However, the mathematical description is simplified if a complex representation is used:

$$
\tilde{E}(t)=\tilde{A}(t) \cdot e^{-i w_{0} t}
$$

where $\tilde{A}(t)$ is the complex envelope, usually chosen such that the real physical field is twice the real part of the 
complex field, and $w_{0}$ is the carrier frequency, usually chosen to the center of the spectrum. In this way the rapidly varying is separated from the slowly varying enveloppe $\widetilde{A}(t) . \widetilde{E}(t)$ can be further decomposed into:

$$
\tilde{E}(t)=|\tilde{E}(t)| \cdot e^{i \varphi_{0}} \cdot e^{-i \varphi(t)}=|\tilde{E}(t)| \cdot e^{i \varphi_{0}} \cdot e^{-i\left(\emptyset(t)-w_{0}\right)}
$$

$\varphi(t)$ is often to as the temporal phase of the pulse and $\varphi_{0}$ the

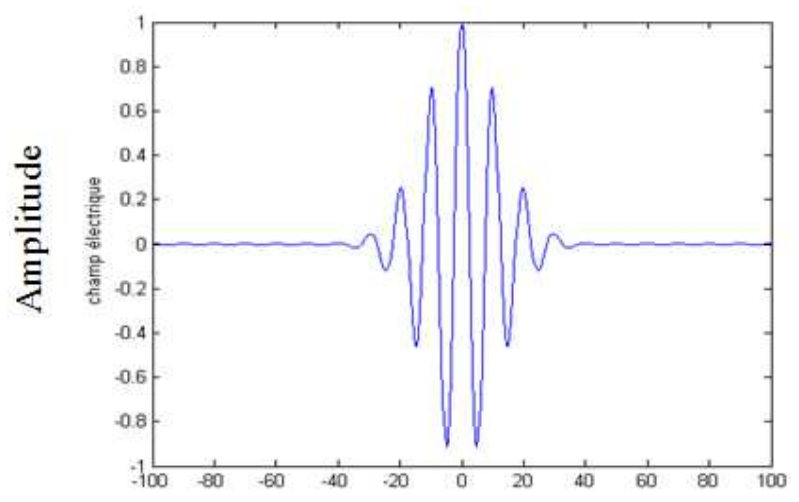

Time in (fs) absolute phase, which relates the position of the carrier wave to the temporal envelope of the pulse (see fig.11). In $\varnothing(t)$ the strong linear term due to the carrier frequency, $w t$, is omitted. which means that a nonlinear temporal phase yields a timedependent frequency modulation- the pulse is said to carry a chirp (illustrated in Fig.11b)

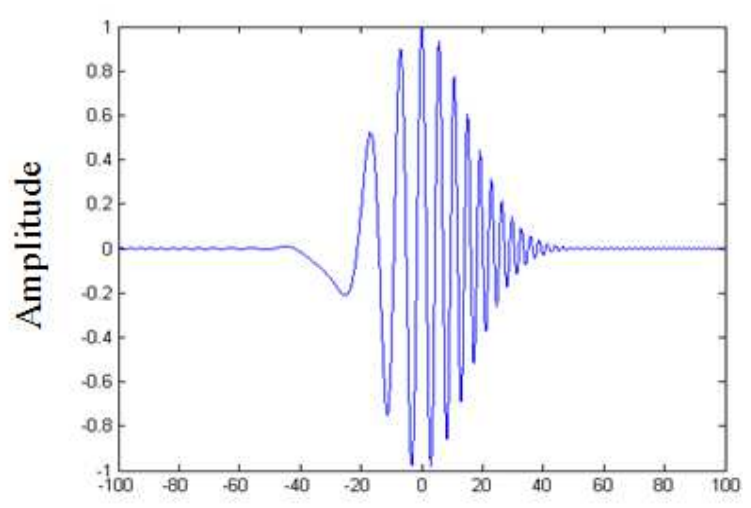

Time in (fs)

Fig 11. (a) The electric field of an ultra-short lasers pulse, (b) The electric field of an ultrashort lasers pulse with a strong positive chirp.

An ultrashort pulse of light will lengthen after it has passed through glass as the index of refraction, which dictates the speed of light in the material, depends nonlinearly on the wavelength of the light. The wavelength of an ultrashort pulse of light is formed from the distribution of wavelengths either side of the center wavelength with the width of this distribution inversely proportional to the pulse duration. Note the frequency variation as a function of time; at the leading edge (to the left) the wavelength is longer than at the trailing edge consisting of only a few cycles.

\section{Phase and chrisp}

Instantaneous phase function of $E(t)$ can be described as the sum of temporal phase and product of carrier frequency with time by the relation $w(t)=\frac{d}{d t} \varnothing(t)+w_{0} t$

Carrier frequency $w_{0}$ has been choosen by minimizing of temporal variation of phase $\varnothing(t)$. The first deriviation of $w(t)$ is defined by temporally-dependent carrier frequency as the result of applying the derivation we receive relation expended in series. Then carrier frequency time denotes quadratic chirp.

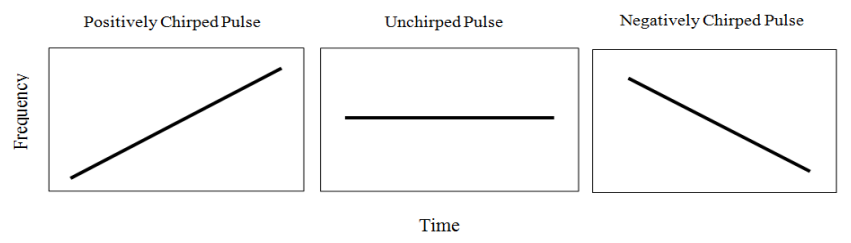

Chirp can be generated due to dispersion of materials they propagate through. Up-chirp is the signal with increasing frequency in time and down-chirp with decreasing frequency in time. Positive chirp is when leading edge of pulse is redshifted in relation to central wavelength and trailing edge is blue-shifted. Negative chirp happens in opposite case. Linear chirp, instantaneous frequency varies linearly with time. The presence of chirp results in significant different delays between the spectrally different components of laser pulse causing pulse broadening effect and leading to a durationbandwidth.

Chirps always appear when ultrashort laser pulses propagate through a medium such as air or glass, where the spectral components of the pulse are subject to a different refractive index. This effect is called Group Velocity Dispersion (GVD). Therefore, for pulse durations on the order of $100 \mathrm{fs}$, the contribution of the quadratic and higher order chirps is negligible. Yet, shorter pulses require the consideration of higher order chirps due to the increasing frequency bandwidth.

\subsubsection{Lens Frequency Domain Description}

It is usually more convenient to represent the pulse in the frequency domain rather than in the time domain. The frequency representation is obtained from the time domain by a complex Fourier transform,

$$
E(w)=\frac{1}{\sqrt{2 \pi}} \int_{-\infty}^{+\infty} E(t) \cdot e^{-i w t} \cdot d t .
$$

Just as in the time domain, $\tilde{E}(w)$ can be written as:

$$
\tilde{E}(w)=|\tilde{E}(w)| e^{i \varphi(w)}
$$

where $\varphi(w)$ now denotes the spectral phase. An inverse transform leads back to the time domain,

$$
\tilde{E}(t)=\frac{1}{\sqrt{2 \pi}} \int_{-\infty}^{+\infty} \tilde{E}(t) \cdot e^{i w t} \cdot d w
$$

From eq. 20 it is clear that $\tilde{E}(t)$ can be seen as a superposition of monochromatic waves. The spectral phase 
can, in the same manner as the temporal phase, be decomposed into different parts. A common procedure is to employ Taylor expansion

$$
\emptyset(w)=\emptyset_{0}+\sum_{n=1}^{\infty} \frac{1}{n !} a_{n}\left(w-w_{0}\right)^{n} \quad \text { with } \quad a_{n}=\left.\frac{d^{n} \varphi}{d w^{n}}\right|_{w=w_{0}}
$$

It can be seen by inserting this Taylor expansion into eq. 21 that the first, two terms will not change the temporal profile of the pulse. A linear phase variation does not change the shape of the pulse, but only introduces a temporal shift of the entire pulse. Therefore, usually only the nonlinear part of the spectral phase is of interest. Any nonlinear addition to the phase will redistribute the frequency components and alter the temporal shape of the pulse.

\subsection{Ultrashort Pulse Measurement Techniques}

Measuring of the pulse width and structure of the complex laser pulses in range under $10 \mathrm{ps}$ is not as routine as the measurement of their repetition rate of wavelength. All techniques for determining the temporal resolution $I(t)$ can be in general classified into direct and indirect methods.

The most commonly used technique of ultrashort laser pulse width measurement is concerned with the study of the temporal intensity profile $I(t)$ through its second-order correlation function that is obtained by the second-harmonic generation. It provides an extremely high time resolution down to tens of femtoseconds. However, being an indirect technique, the intensity autocorrelation cannot provide unequivocal information on the pulse shape and, in particular, its asymmetry. Strictly speaking, higher order correlation functions are necessary for the exact determination of the pulse shape and duration. On the other hand, when performed with interferometric accuracy, second - order autocorrelations provide a much more contrasted pattern and information about the pulse shape and chirp may be extracted. However all techniques employ principle of probing ultrashort pulse with time delayed replica of pulse itself. Indirect methods can be distinguished into self - referenced and nonself-referenced, interferometric or non-interferometric. According to pulse retrieval that can be direct (non-iterative) or indirect (iterative) and the data can be captured and analyzed in the real time or not. Ultrashort - pulse characterization techniques, such as the numerous variants of frequency resolved optical gating (FROG) and spectral phase interferometry for direct electricfield reconstruction (SPIDER), fail to fully determine the relative phases of well separated frequency components. If well-separated frequency components are also characterized gate pulses are used (Joanna, 2007).

\subsubsection{Non-Interferometric Techniques}

\subsubsection{Intensity Autocorrelation}

Complex electric field $E(t)$ corresponds to intensity $I(t)=|E(t)|^{2}$ and an intensity autocorrelation function is defined by

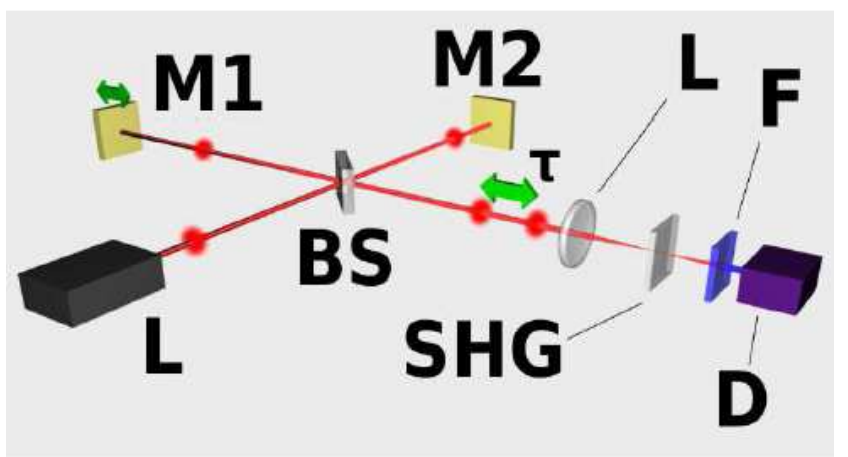

Fig 12. Setup for an interferometric autocorrelator, similar to the field autocorrelator above, with the following optics added: L: converging lens, SHG: second-harmonic generation crystal, F: spectral filter to block the fundamental wavelength.

$$
A(\tau)=\int_{-\infty}^{+\infty} I(t) I(t+\tau) d t
$$

Two parallel beams with a variable delay are generated, then focused into a second-harmonic-generation crystal to obtain a signal proportional to $E(t)+E(t+\tau$. Only the beam propagating on the optical axis, proportional to the cross product $E(t) E(t-\tau)$ is retained. This signal is then recorded by a slow detector, which measures

$$
I(\tau)=\int_{-\infty}^{+\infty}|E(t) E(t-\tau)|^{2} d t=\int_{-\infty}^{+\infty} I(t) I(t-\tau) d t
$$

$I(\tau)$ is exactly the intensity autocorrelation $A(\tau)$. Both beams must be focused at the same point inside the crystal as the delay is scanned in order for the second harmonic to be generated. It can be shown that the intensity autocorrelation width of a pulse is related to the intensity width. For a Gaussian time profile, the autocorrelation width is longer than the width of the intensity, and it is 1.54 longer in the case of an hyperbolic secant squared (sech2) pulse. This numerical factor, which depends on the shape of the pulse, is sometimes called the deconvolution factor. If this factor is known, or assumed, the time duration (intensity width) of a pulse can be measured using an intensity autocorrelation. However, the phase cannot be measured (Joanna, 2007), (abdolah, 2007).

From the construction of the setup point of view as intensity autocorrelation as also interferrometric autocorrelation consist of the some components, only the geometry and processing the captured data is different. In case of intensity autocorrelation is applied non-collinear geometry and in case of interferrometric setup the collinear geometry of beams incomming to the nonlinear medium.

\subsubsection{FROG}

The technique of Frequency-Resolved Optical Gating (FROG) has been introduced by Trebino and coworkers. In FROG technique signal $E_{1}$ has been temporally shifted about $\tau$ through time-delay element in respect with signal $E_{2}$. Then, two signals have been in nonlinear medium noninterferometrically overlapped. As the result of SFG or DFG process (at the efficient phase matching conversion) one receive the FROG signal. 


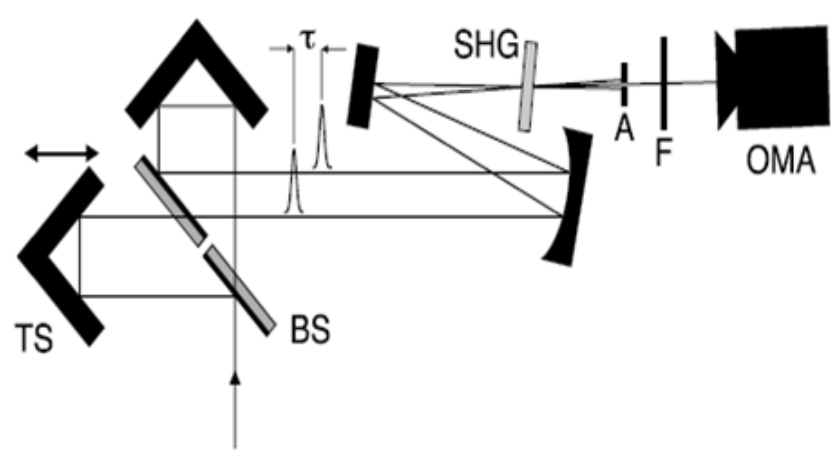

Fig 13. FROG reconstruction scheme. When both $E_{1}$ and $E_{2}$ have been unknown, then we deal with Blind-FROG problem. When $E_{1}=E_{2}$ then we have to do with SHG-FROG problem.

In construction with process II also collinear geometry is possible.

$$
\begin{gathered}
E_{F R O G}(\Omega, \tau) \propto \int_{-\infty}^{+\infty} E_{1}(t-\tau) E_{2}(t) \exp (i \Omega t) d t \\
\propto \int_{-\infty}^{+\infty} \tilde{E}_{1}(w) \tilde{E}_{2}(\Omega-w) \exp (i w \tau) d w \\
\propto \int_{-\infty}^{+\infty} d t \int_{-\infty}^{+\infty} \tilde{E}_{1}(w) E_{2}(t) \exp (-i w t) \exp (i \Omega t+i w \tau) d w
\end{gathered}
$$

The spectral intensity

$$
I_{F R O G}(\Omega, \tau) \propto\left|E_{F R O G}(\Omega, \tau)\right|^{2}
$$

has been at the various $\tau$ by spectrometer measured and $I_{F R O G}(\Omega, \tau)$ is called FROG-trace. Relations (25), (26) are only the mathematical representation of eq.24. The task of receiving unknown complex signals $E_{1}$ and $E_{2}$ from measured FROG trace is known as the FROG reconstruction problem

FROG involves measuring the spectrum of the signal pulse as a function of the delay between two input pulses. The resulting trace of intensity versus frequency and delay is related to the pulse's spectrogram, a visually intuitive transform containing both time and frequency information. Using phase retrieval concepts that the FROG trace yields the full intensity $I(t)$ and phase $\emptyset(t)$ of an arbitrary ultrashort pulse. As has been already mentioned, several schemes and methods exist for frequency-resolved optical gating as a technique for the full characterization of ultrashort optical signals as complex electric fields. However, the uniqueness of the reconstructed fields has never been shown. There exist no proof of PCGP algorithm and some papers showing a lot of trivial and non-trivial ambiguities, but going into the details it depends on the applied conditions (Greg, 1996),(Marco, 1996).

\subsubsection{Interferometric Techniques}

\subsubsection{Interferometric Autocorrelation}

Setup for an interferometric autocorrelator is similar to the field autocorrelator above, with the following optics added: L: converging lens, SHG: second harmonic generation crystal, F: spectral filter to block the fundamental wavelength. A nonlinear crystal can be used to generate the second harmonic at the output of a Michelson interferometer in a collinear geometry. In this case, the signal recorded by a slow detector is

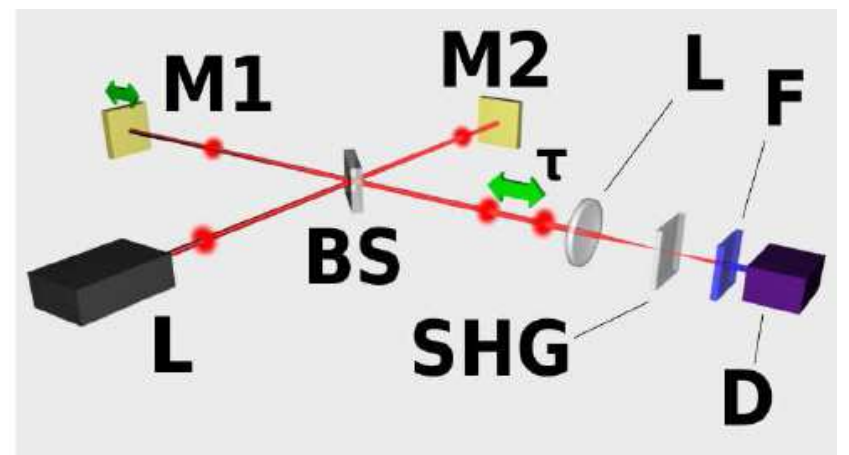

Fig 14. Setup for an interferometric autocorrelator, similar to the field autocorrelator above, with the following optics added: L: converging lens, SHG: second-harmonic generation crystal, F: spectral filter to block the fundamental wavelength.

$$
(\tau)=\int_{-\infty}^{+\infty}|E(t)+E(t-\tau)|^{2} d t
$$

$I(\tau)$ is called the interferometric autocorrelation. It contains some information about the phase of the pulse: the fringes in the autocorrelation trace wash out as the spectral phase becomes more complex

\subsubsection{SPIDER}

The Spectral Phase Interferometry for Direct Electric-field Reconstruction technique (SPIDER) is based on spectral interferometry and needs no components which has to be shifted over the measurement process. From the signal $E(t)$ that should be characterized, the copy-signal is being generated by beam splitter

$$
E(t-\tau) \exp \left[i w_{0} \tau\right]
$$

The time between the signal and copy itself has been established through fixed position at the optical delay-line. Then the copy of signal goes through phase filter (dispersive medium, for instance SF10 glass), so arises the signal

$$
E_{M}(t)=F^{-1}\left\{\tilde{E}(w) \exp \left[i \emptyset_{M}(w)\right]\right\}
$$

Through the phase filter electric field $\tilde{E}(w)$ get additional spectral phase $\emptyset_{M}(w)$, which corresponds to temporal extension $E(t)$. From the signals

$$
E_{M}(t) \text { and } E(t-\tau) \exp \left[i w_{0} \tau\right]+E(t)
$$

SFG-Signal can be created

$$
\begin{gathered}
E_{S F G}(t) \propto E_{M}(t)\left\{E(t-\tau) \exp \left[i w_{0} \tau\right]+E(t)\right\} \\
\quad=E_{M}(t) E(t-\tau) \exp \left[i w_{0} \tau\right]+E_{M}(t) E(t)
\end{gathered}
$$

As square law detectors are not sensitive to the phase, the measurement of the intensity (whether it is spatial or spectral) is an easy task but the measurement of the phase needs indirect solutions. Spectral shearing is similar in concept to intensity autocorrelation except that, instead of gating pulse 
with a time-shifted copy of itself, a pulse is interfered with frequency-shifted or spectrally sheared copy of itself. SPIDER uses nonlinear mixing (usually SFG) as combination of filters to generate signal that can be measured by slow detector.

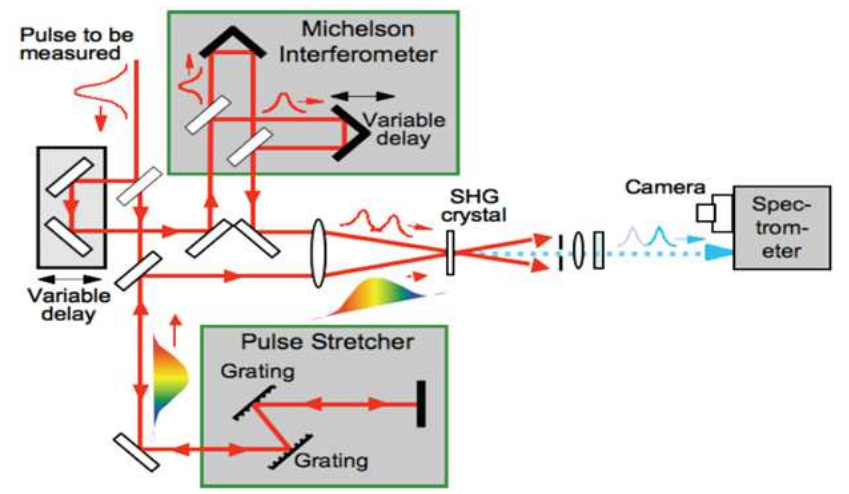

Fig 15. Main steps of SPIDER technique.

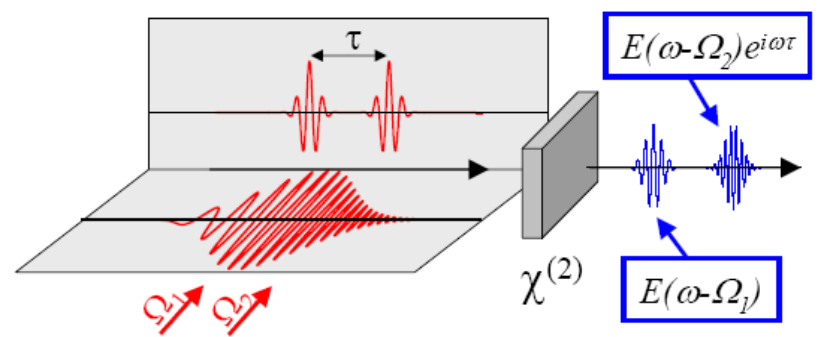

Fig 16. generation of two sheared replicas of the input pulse by non-linear interaction with a chirped pulse

Sending through linear spectral phase modulator (adds spectral shear to pulses in its arm of interferometer). Sending through linear temporal modulator - adds temporal delay to the pulse in its arm, then two pulses are recombined and send through a spectrometer. Spectral interferometry allows us obtain difference between two spectral phases. To the spectral interferometry spectrum one should apply fast Fourier transform and as the product one will achieve in form of one center peak and two sidebands lower peaks in the time domain.

Centered peak contains only spectrum information. One filter out two peaks and from existing one can receive spectral phase difference by applied inverse fast Fourier transform. To the main advantages of the SPIDER method can be counted following properties: pulse retrieval is direct (non-iterative), minimal data are required: only one spectrum yields spectral phase. It naturally operates single-shot. Disadvantages: apparatus is very complicated (13 alignment parameters). and unability to measure long and complex pulses, the temporal delay $\tau$ should be chosen very accurate, also the zeros in complex pulses make a problems. In stretched pulse we have continuous waves approximately, so there are usually weak pulses, sampling, aliasing effects in Fourier Transforms when would be too much fringes and if would be too not enough fringes then is not good resolved, so this issue needs a compromise (Hofmann, 2005).

\subsubsection{The VAMPIRE Technique}

Abbreviation VAMPIRE means: Very Advanced Method of Phase and Intensity Retrieval of E-fields. The optical scheme of idea of VAMPIRE technique is shown in Fig.17 below. One can classified VAMPIRE as the noninterferometric spectrographic technique. The conditioning filter is a temporal phase filter and a key element of VAMPIRE. Every spectrogram generated by VAMPIRE has special mathematical properties. For that reason a unique retrieval of two independent and arbitrary ultrafast pulses is possible. Every spectrogram generated by Vampire has special mathematical properties. For that reason a unique retrieval of two independent and arbitrary ultrahort pulses is possible (Joanna, 2007).

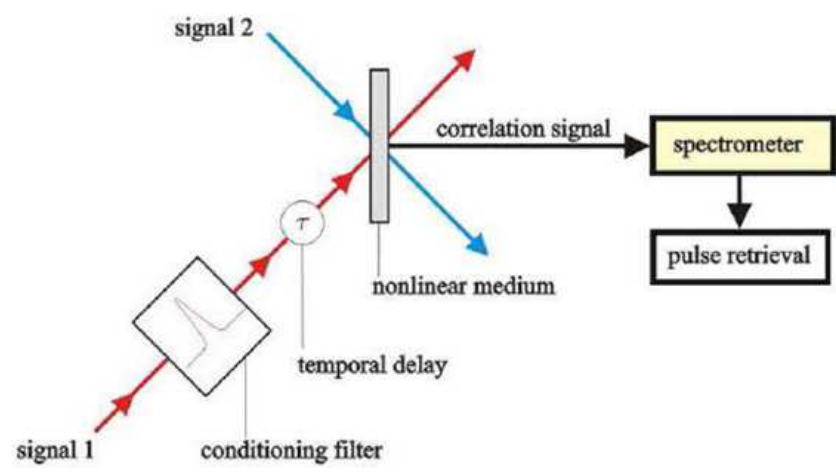

Fig 17. General view on idea of VAMPIRE technique

Ultrashort pulses with well-separated multiple frequency components cannot be retrieved by all variants of selfreferenced FROG or self-referenced SPIDER. Ultrashort signals with well-seperated multiple frequency components are no problem for VAMPIRE. Complex ultrashort signals cannot be retrieved by SHG- FROG, PG - FROG or Blind FROG in general. Reconstruction scheme is presented below. The main features of VAMPIRE technique can be listed as follows

- Unique retrieval of two independent and arbitrary ultrafast pulses;

- Very fast real-time retrieval algorithm without stagnation problems;

- Pulse width range: from $10 \mathrm{fs}$ to $1 \mathrm{ps}$;

- Wavelength range: depends on the given pulse, in the Vampire model a wide range from UV to IR is applicable;

- Input energy requirements: $10 \mathrm{~mW}$ average power or $50 \mu \mathrm{J}$ single pulse (but depends on the two signals in general);

- Easy alignment.

All existing techniques usually present the good results by analysis simple pulse shapes. More complex amplitude profiles become problematic, especially when there are zeroes or well separated parts in temporal or spectral domain. In such experiments, iterative FROG algorithm often does not converge and give wrong output. Therefore, in case of more complex signals full field reconstruction could not be guaranteed by conventional FROG methods. VAMPIRE 
method is derived from Blind-FROG and is based not on autocorrelation of the signal, but rather on its crosscorrelation with suitable reference pulse. Signal and reference are also known as probe pulse and gate pulse. The VAMPIRE spectrogram (strictly speaking, spectrochronogram) can be expressed by

$$
I(\Omega, \tau) \propto\left|\int \tilde{E}_{\text {gate }}(w) \tilde{E}_{\text {probe }}(\Omega-w) \exp (i w \tau) d w\right|^{2}
$$

where $\tilde{E}$ is the complex spectral field, $\Omega$ defines the specrtal axis, and $\tau$ is the delay. Vampire can guarantee the uniqueness of full field reconstruction because $\tilde{E}_{\text {gate }}$ can be chosen such that $I(\Omega, \tau)$ does not suffer from non-trivial ambiguities. Ideally, this would be achieved by a wellseparated double pulse structure with an asymmetry in duration, peak power, and chirp of the individual pulses.

A sufficient symmetry is required, but detailed knowledge of the structure is definitely not. In VAMPIRE spectrogram exists non-centrosymmetric geometry due to existing of two spectrally dispersed signals from the cross-correlations of the probe pulse with two different components of the gate pulse. In comparison to autocorrelation based techniques, this asymmetry in the cross-correlation represents just that extra amount of information that allows a unique relation between probe pulse and spectrogram.

\section{Propagation of a Light Pulse in a Transparent Medium}

What happens to a short optical pulse propagating in a transparent medium? Because of its wide spectral width and because of group velocity dispersion in transparent media, it undergoes a phase distortion inducing an increase of its duration (Khelladi, 2005).

When an electromagnetic wave interacts with the bound electrons of a dielectric medium the medium response in general depends on the optical frequency of the wave. This property is referred to as chromatic dispersion. It manifests itself through frequency dependence of the refractive index of the medium. Its origin is related to the characteristic resonance frequencies at which the medium absorbs the electromagnetic radiation through the oscillation of the bound electron. Far from resonances, the refractive index of the medium is well approximated by the Sellmeier equation. The frequency Fourier transform of a Gaussian pulse has already been given as

$$
E(w)=\exp \left(\frac{-\left(w-w_{0}\right)^{2}}{4 . \Gamma}\right)
$$

An ultrashort Fourier limited pulse has a broad spectrum and no chirp; when it propagates a distance through a transparent medium, the medium introduces dispersion to the pulse inducing an increase in the pulse duration. To investigate and determine the dispersion, we assume a Gaussian shape for the pulse. The electric field of the pulse is given as Eq.35

After the pulse has propagated a distance $z$, its spectrum is modified to

$$
E(w, z)=E(w) \exp [ \pm i k(w) z], k(w)=\frac{n(w) \cdot w}{c}
$$

where $\mathrm{k}(\mathrm{w})$ is now a frequency-dependent propagation factor. In order to allow for a partial analytical calculation of the propagation effects, the propagation factor is rewritten using a Taylor expansion as a function of the angular frequency, assuming that $\Delta w \ll w_{0}$ (this condition is only weakly true for the shortest pulses). Applying the Taylor expansion to eq.37, the pulse spectrum becomes.

$$
\begin{aligned}
& k(w)=k\left(w_{0}\right)+k^{\prime}\left(w-w_{0}\right)+\frac{1}{2} k^{\prime \prime}\left(w-w_{0}\right)^{2}+(37) \\
& \text { where } k^{\prime}=\left(\frac{d k(w)}{d w}\right)_{w_{0}} \text {. and } k^{\prime \prime}=\left(\frac{d^{2} k(w)}{d w^{2}}\right)_{w_{0}}, \\
& \qquad E(w, z)=\exp \left[-i k\left(w_{0}\right) z-i k^{\prime} z\left(w-w_{0}\right)-\left(\frac{1}{4 \Gamma}+\right.\right. \\
& i 2 k^{\prime \prime}(w-w 0) 2
\end{aligned}
$$

The time evolution of the electric field in the pulse is then derived from the calculation of the inverse Fourier transform of eq.39,

$$
e(t, z)=\int_{-\infty}^{+\infty} E(w, z) \cdot e^{-i w t} d w
$$

so that

$$
e(t, z)=\sqrt{\frac{\Gamma(z)}{\pi}} \cdot \exp \left[i w_{0}\left(t-\frac{z}{V_{\emptyset}\left(w_{0}\right)}\right)\right] \times \exp \left[-\Gamma(z)\left(t-\frac{z}{V_{g}\left(w_{0}\right)}\right)^{2}\right]
$$

where

$$
V_{\varnothing}\left(w_{0}\right)=\left(\frac{w}{k}\right)_{w_{0}}, \quad V_{g}\left(w_{0}\right)=\left(\frac{d w}{d k}\right)_{w_{0}}, 1 /\left(\Gamma(z)=1 / \Gamma+2 i k^{\prime \prime} z\right.
$$

In the first exponential term of eq.40, it can be observed that the phase of the central frequency $w_{0}$ is delayed by an amount $\frac{z}{V_{\varnothing}}$ after propagation over a distance $z$. Because the phase is not a measurable quantity, this effect has no observable consequence. The phase velocity $V_{\varnothing}\left(w_{0}\right)$ measures the propagation speed of the plane wave components of the pulse in the medium. These plane waves do not carry any information, because of their infinite duration.

The second term in eq.40 shows that, after propagation over a distance $z$, the pulse keeps a Gaussian envelope. This envelope is delayed by an amount $z / V_{g}, V_{g}$ being the group velocity. The second term in eq. 40 also shows that the pulse envelope is distorted during its propagation because its form factor $\Gamma(z)$, defined as

$$
1 /\left(\Gamma(z)=1 / \Gamma+2 i k^{\prime \prime} z\right.
$$

Depends on the angular frequency $w$ through $k^{\prime \prime}(w)$,

$$
k^{\prime \prime}=\left(d^{2} k / d w^{2}\right)_{w_{0}}=\frac{d}{d w}\left(\frac{1}{v_{g}}\right)_{w_{0}}
$$

This term is called the "Group Velocity Dispersion". The temporal width of the pulse at point $\mathrm{z}$ : 


$$
\Delta \tau_{z}=\Delta \tau_{0} \sqrt{1+4 \cdot\left(\Gamma \cdot k^{\prime \prime} z\right)^{2}} .
$$

with $k^{\prime \prime}=\frac{\lambda^{3}}{2 \cdot \pi \cdot c^{2}} \frac{d^{2} n}{d \lambda^{2}} \Gamma=\frac{2 \log 2}{\Delta_{0}^{2}}$,

\subsection{Application in Litharge Index SF57 Medium}

In optical materials, the refractive index is frequency dependent. This dependence can be calculated for a given material using a Sellmeier equation, typically of the form

$$
n^{2}(w)=1+\sum_{i=1}^{m} \frac{B_{i} w_{i}^{2}}{w_{i}^{2}-w^{2}}
$$

The glass containing lead-oxide $(\mathrm{PbO})$ are called Litharge glasses. Litharge has a high refractive index making it bright and a relatively soft surface making it easy to decorate by grinding, cutting and engraving. Its ability to refract light more efficiently than standard glass makes it an excellent material for prisms, decorative objects, and artificial jewelry. It is also favoured for electrical applications because of excellent electrical insulating characteristics, and the concave or diverging half on an achromatic lens is usually made of litharge glass.

Lead-silicate glasses are especially promising materials for highly nonlinear fibers due to their suitable combination of properties. Although their material nonlinearities are lower than those of bismite, tellurite, and chalcogenide glasses, they provide higher thermal and crystallization stability and less steep viscosity temperature curves, while exhibiting low softening temperatures. The properties of $\mathrm{PbO}$ based glasses are summarized below.

- High linear refractive index (1.75-2.4)

- High nonlinear index coefficient $\left(22-286.10^{-20}\left[\mathrm{~m}^{2} / \mathrm{W}\right]\right)$

- Wide transmission range $(0.4 \mu \mathrm{m} \sim 3 \mu \mathrm{m})$

- Transition temperature $\left(\sim 700^{\circ} \mathrm{C}\right)$ and melting temperature $\left(\sim 1100^{\circ} \mathrm{C}\right)$

- Poor electrical conductivity

- Highest atomic number of all stable elements The index of litharge SF57 is given by the following expression (45):

Table 1. Parameters for litharge SF57 Glasses.

\begin{tabular}{clll}
\hline$B_{i}$ & 1.81651732 & 0.428893631 & 1.07186278 \\
$\lambda_{i}(\mu \mathrm{m})$ & 0.0143704198 & 0.0592801172 & 121.419942 \\
\hline
\end{tabular}

where $w_{i}$ is the frequency of resonance and $B_{i}$ is the amplitude of resonance. In the case of optical fibers, the parameters $w_{i}$ and $B_{i}$ are obtained experimentally by fitting the measured dispersion curves to eq. 45 with $m=3$ and depend on the core constituents (Jong Kook, 2005).

\subsection{Parameter of Dispersion}

An ultrashort Fourier limited pulse has a broad spectrum and no chirp; when it propagates a distance through a transparent medium, the medium introduces dispersion to the pulse inducing an increase in the pulse duration. We consider dispersions of orders two. The pulse broadens on propagation as a result of group velocity dispersion (GVD).

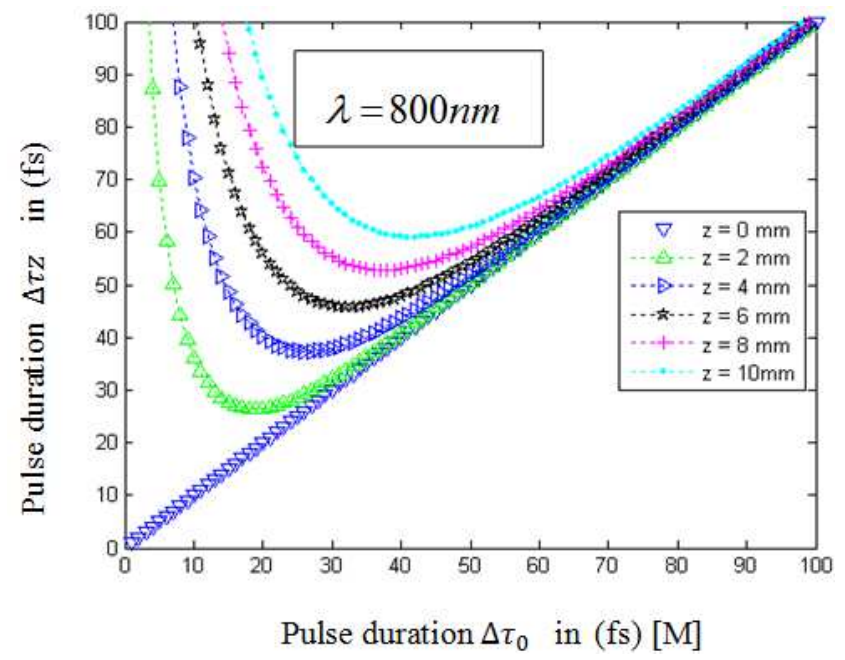

Fig 18. Temporal broadening of the transform-limited pulse for different values of the propagation distance $z$.

In summary, the propagation of a short optical pulse through transparent medium results in a delay of the pulse, a duration broadening and a frequency chirp.

\subsection{Group Velocity Dispersion}

The Group Velocity Dispersion (GVD) is defined as the propagation of different frequency components at different speeds through a dispersive medium. This is due to the wavelength-dependent index of refraction of the dispersive material. GVD causes variation in the temporal profile of the laser pulse, while the spectrum remains unaltered.

To the first place we limited only to the order two of the Taylor expansion of the phase. It is noticed that the analysis of Fourier remains valid only for durations of pulse which are higher than $\approx 60$ fs.

In addition media we consider all higher order dispersion, which completely describes the physical processes involved in ultrashort dispersive pulse dynamics. The pulse broadens in time and becomes asymmetric. In addition, the off axis pulse becomes wider than the pulse on axis (Khelladi, 2008).

$$
\left.\varphi(w)=\varphi\left(w_{0}\right)+\left(w-w_{0}\right) \frac{d \varphi}{d w}\left|+\frac{1}{2 !}\left(w-w_{0}\right)^{2} \frac{d^{2} \varphi}{d w^{2}}\right|_{w=w_{0}}+\cdots+\left.\frac{1}{n !}\left(w-w_{0}\right)^{n} \frac{d^{n} \varphi}{d w^{n}}\right|_{w=\Omega}+\theta(w)\right)_{w=w_{0}}
$$




$$
\left\{\begin{array}{c}
\varphi(\lambda)=\frac{2 \pi}{\lambda} n(\lambda) z \\
\frac{d \lambda}{d w}=-\frac{\lambda^{2}}{2 \pi c} \\
\frac{d \varphi}{d w}=-\frac{z}{c}\left[\frac{d n}{d \lambda}-n\right] \\
\frac{d^{2} \varphi}{d w^{2}}=+\frac{\lambda^{3}}{4 \pi^{3} c^{2}} \frac{d^{2} n}{d \lambda^{2}} z \\
\frac{d^{3} \varphi}{d w^{3}}=-\frac{\lambda^{4}}{4 \pi^{2} c^{3}}\left[3 \frac{d^{2} n}{d \lambda^{2}}+\lambda \frac{d^{3} n}{d \lambda^{3}}\right] Z \\
\frac{d^{4} \varphi}{d w^{4}}=+\frac{\lambda^{5}}{8 \pi^{3} c^{4}}\left[12 \frac{d^{2} n}{d \lambda^{2}}+8 \lambda \frac{d^{3} n}{d \lambda^{3}}+\lambda^{2} \frac{d^{4} n}{d \lambda^{4}}\right] Z \\
\frac{d^{5} \varphi}{d w^{5}}=-\frac{\lambda^{6}}{16 \pi^{4} c^{5}}\left[60 \frac{d^{2} n}{d \lambda^{2}}+60 \lambda \frac{d^{3} n}{d \lambda^{3}}+15 \lambda^{2} \frac{d^{4} n}{d \lambda^{4}}+\lambda^{3} \frac{d^{5} n}{d \lambda^{5}}\right] Z \\
\frac{\lambda^{6} \varphi}{d w^{6}}=+\frac{d^{3}}{32 \pi^{5} c^{6}}\left[360 \frac{d^{2} n}{d \lambda^{2}}+480 \lambda \frac{d^{3} n}{d \lambda^{3}}+180 \lambda^{2} \frac{d^{4} n}{d \lambda^{4}}+24 \lambda^{3} \frac{d^{5} n}{d \lambda^{5}}+\lambda^{4} \frac{d^{6} n}{d \lambda^{6}}\right] Z
\end{array}\right.
$$

$$
\left[\begin{array}{l}
\emptyset^{(2)} \\
\emptyset^{(3)} \\
\emptyset^{(4)} \\
\emptyset^{(5)} \\
\emptyset^{(6)}
\end{array}\right]=(-1)^{n} 2 . \pi \cdot z\left[\frac{\lambda}{2 . \pi \cdot c}\right]^{n}\left[\begin{array}{lllll}
1 & 0 & 0 & 0 & 0 \\
3 & 1 & 0 & 0 & 0 \\
12 & 8 & 1 & 0 & 0 \\
60 & 60 & 15 & 1 & 0 \\
360 & 480 & 180 & 24 & 1
\end{array}\right]
$$

It seems to me that we can write $\varphi^{(i)}=\frac{d^{i} \varphi}{d w^{i}}$ as a recurrence, giving $\varphi^{(i)}$ based on derivatives of order $i$, the index of refraction. Matrix form, we can write

$$
\begin{gathered}
\emptyset(w)=\emptyset\left(w_{0}\right)+\left(w-w_{0}\right) \emptyset^{(1)}+\left.\sum_{i=2}^{p} \frac{1}{i !}\left(w-w_{0}\right)^{i} \emptyset^{(i)}\right|_{w=w_{0}}+\theta(w) . \\
\emptyset^{(p)}=(-1)^{p} .2 \pi . z\left[\frac{\lambda}{2 . \pi \cdot c}\right]^{p} \sum_{j=2}^{p} \lambda^{j-1} A(p-1, j-1) n^{(j)} \text { with } p>2
\end{gathered}
$$
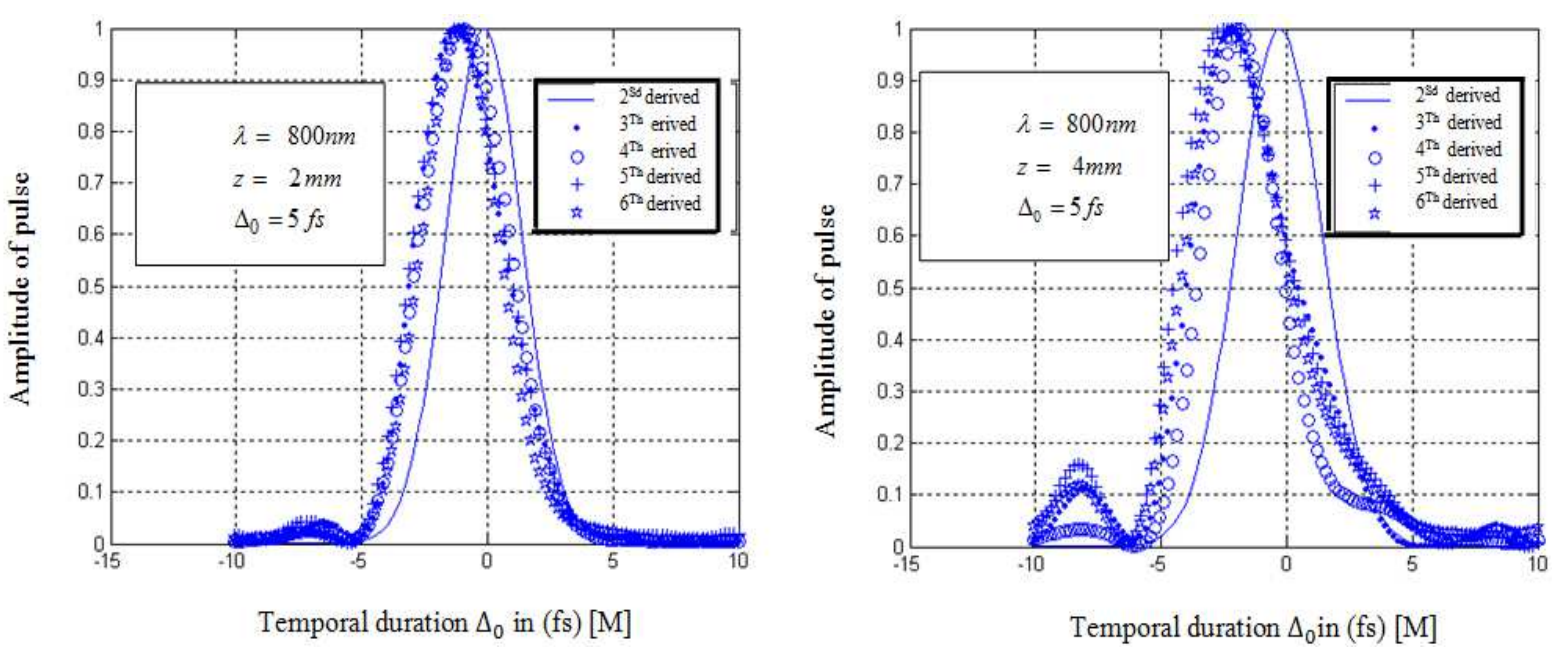

Fig 19. (a) the pulse broadens on propagation as a result of group velocity dispersion (GVD) (b) The pulse shape is no longer Gaussian and it becomes asymmetric due to higher order dispersions

Analytically known and experimentally observed propagation affects such as spectral shift, pulse broadening and asymmetry in dispersive media can be easily brought out in the simulation using formalism presented here. In addition, such studies can be extended to pulses of arbitrary temporal shape without any further algorithmic complexity by numerical simulation. Higher order dispersion effects can be handled easily in the numerical simulation unlike in the case of analytical calculation (Khelladi, 2008).

Discussion

The Fourier theorem is the most classical approach for describing the propagation of electromagnetic signals 
through dispersive media. In the case of signals characterized by a slow temporal varying envelope, the phase is usually approximated by the Taylor expansion in the neighborhood of the central frequency of the input pulse. For shorter pulses, the concept of group velocity is irrelevant and the envelope distortion is a function of the higher order terms. Ultrashort pulses less than $10 \mathrm{fs}$ are now available. Their envelope harmonic content is so high that the Taylor expansion of the phase is now more possible. There is no other way than a numerical computation of the Fourier integral.

However this method does not permit a straightforward physical understanding of the envelope propagation and principally does not picture the fact that this is the group velocity dispersion which generates the ultrashort pulses distortions. Such a situation claims for another type of decomposition involving both a time and frequency dependence of the components.

Numerous bidimensional representations of acoustic and electromagnetic signals have already been suggested. We propose here a method derived from the Gabor transformation in order to decompose the signal into an infinite number of elementary components (wavelet) of same duration (much more longer than that of the original signal), each of them being centered at a frequency $\Omega$ belonging to the Fourier spectrum of the pulse (Khelladi, 2008).

\section{Time-Frequency Decomposition}

\subsection{Wavelet Theory}

The wavelets are very particular elementary functions, these are the shortest vibrations and most elementary that one can consider. One can say that the wavelet east carries out a zooming on any interesting phenomenon of the signal which place on a small scale in the vicinity of the point considered (Meyer et al, 1987).

\subsection{Wavelet Techniques}

Starting with a signal $e(t)$, in plane $z=0$, we define wavelet centered at $\Omega$ by

$$
\begin{gathered}
\theta(\Omega)=E(w) \cdot \exp \left[-\frac{(w-\Omega)^{2}}{4 \gamma}\right], \quad \text { with } E(w)= \\
\frac{E_{0}}{2 \cdot \pi} \sqrt{\frac{\pi}{\Gamma}} \exp \left[\frac{\left(w-w_{0}\right)^{2}}{4 \cdot \Gamma}\right]
\end{gathered}
$$

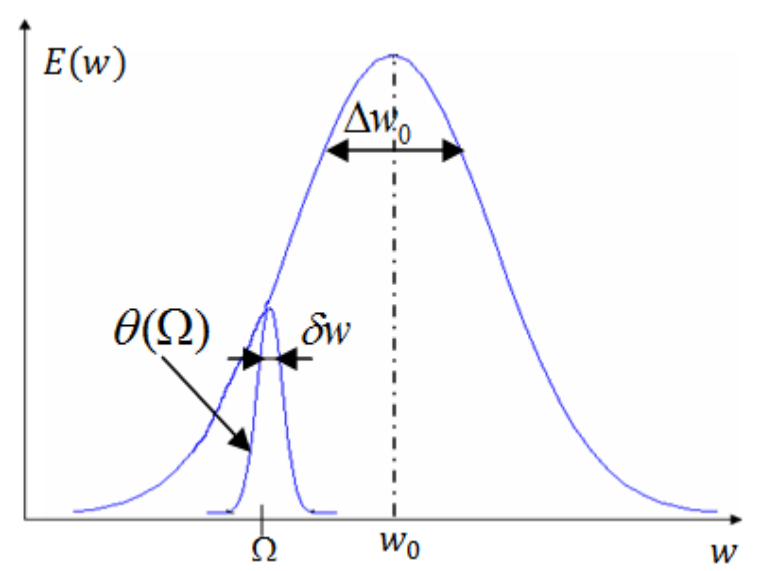

Fig 20. Gaussian envelope decomposed on a number of wavelet we calculates the electric field associated with the wavelet $\theta(\Omega, z=0)$.

$$
\theta(t, z=0)=T F\{\theta(\Omega, z=0)\}
$$

$$
\theta(t, z=0)=E_{0} \sqrt{\frac{\gamma}{\gamma+\Gamma}} \cdot \exp \left[\frac{-\left(w_{0}-\Omega\right)^{2}}{4(\gamma+\Gamma)}\right] \cdot \exp \left[-\frac{\gamma \Gamma}{\gamma+\Gamma} t^{2}\right] \cdot \exp \left[j \frac{\gamma w_{0}+\Gamma \Omega}{\gamma+\Gamma} t\right]
$$

- In time, the pulse is also Gaussian, of parameter $\frac{\gamma \Gamma}{\gamma+\Gamma}$.

- The maximum of amplitude of the wavelet $\theta(t, z=0)$ vary with $\Omega$, center frequency of analysis on Gaussian of parameter $\gamma+\Gamma$.

- The signal propagates in the positive $z$ direction in a linear dispersive and transparent medium, which fills the half space $z>0$ and whose refractive index is $n(w)$. After propagation, the wavelet $\theta(\Omega, x)$ may be written

$$
\theta(\Omega, z)=\frac{E_{0}}{2 \cdot \sqrt{\pi} \gamma} E(w) \cdot \exp \left[-\frac{(w-\Omega)^{2}}{4 \gamma}\right] \cdot \exp [j \emptyset(w)] \text {. }
$$

As already mentioned, $\tau_{\text {wavelet }}$ is large enough to ensure that analyzing function has only non negligible values over a spectral range lying in the neighbourhood of $\Omega$ in (Fig 20). Under these circumstances, we have

$$
\emptyset(w)=\emptyset(\Omega)+(w-\Omega) \frac{d \emptyset}{d w}\left|+\frac{1}{2 !}(w-\Omega)^{2} \frac{d^{2} \emptyset}{d w^{2}}\right|_{w=\Omega}+\cdots+\left.\frac{1}{n !}(w-\Omega)^{n} \frac{d^{n} \emptyset}{d w^{n}}\right|_{w=\Omega}+\theta(w)_{\cdot w=\Omega}
$$

Neglecting the higher terms in eq.50:

$$
\begin{gathered}
\emptyset(w)=\emptyset(\Omega)+\left.(w-\Omega) \frac{d \emptyset}{d w}\right|_{w=\Omega}+\left.\frac{1}{2 !}(w-\Omega)^{2} \frac{d^{2} \emptyset}{d w^{2}}\right|_{w=\Omega}+\theta(w) . \\
\theta(\Omega, z)=\frac{E_{0}}{2 \cdot \sqrt{\pi} \gamma} \sqrt{\frac{\pi}{\Gamma}} \exp \left[-\frac{\left(w-w_{0}\right)^{2}}{4 \Gamma}\right] \cdot \exp \left[-\frac{(w-\Omega)^{2}}{4 \gamma}\right] \cdot \exp \left[j \emptyset^{(0)}+j(w-\Omega) \emptyset^{(1)}+\frac{1}{2} j(w-\Omega)^{2} \cdot \emptyset^{(2)}\right] \\
\theta(t, z)=\frac{1}{2 \pi} \int_{-\infty}^{+\infty} \theta(\Omega, z) \cdot \exp (j w t) d w
\end{gathered}
$$

We calculates the temporal electric field associated with the wavelet $\theta(\Omega, z)$. 


$$
\begin{array}{r}
\theta(t, z)=\frac{1}{2 \pi} \frac{E_{0}}{2 \cdot \sqrt{\pi} \gamma} \sqrt{\frac{\pi}{\Gamma}} e^{\left[-\frac{\left(\Omega-w_{0}\right)^{2}}{4 \Gamma}\right]} e^{\left(j \emptyset^{(0)}\right)} \times e^{-\left[\frac{1}{4 \Gamma}+\frac{1}{4 \gamma}-\frac{1}{2} j \emptyset^{(2)}\right] \Omega^{2}} \cdot e^{\left[\frac{\left(\Omega-w_{0}\right)}{2 \Gamma}-j \emptyset^{(1)}\right] \Omega} \\
\times\left(\int_{-\infty}^{+\infty} e^{-\left[\frac{1}{4 \Gamma}+\frac{1}{4 \gamma}-\frac{1}{2} j \emptyset^{(2)}\right] w^{2}} \cdot e^{\left[\frac{1}{4 \Gamma}+\frac{1}{4 \gamma}-\frac{1}{2} j \emptyset^{(2)}\right] 2 \Omega w} \times e^{\left[-\frac{\left(\Omega-w_{0}\right)^{2}}{2 \Gamma}-j \emptyset^{(1)}\right]} \cdot e^{j w t}\right) d w
\end{array}
$$

The amplitude of the incident $\Omega$ wavelet is given from eq. 60 by

$$
\begin{aligned}
& \theta(t, z)=\frac{E_{0}}{2 \cdot \sqrt{\pi} \gamma} \sqrt{\frac{\Gamma(z)}{\Gamma}} \cdot \exp \left(j \emptyset^{(0)}\right) \exp \left(-\Gamma(z)\left[t+\frac{z}{V_{g}(\Omega)}\right]^{2}\right) \\
& \times \exp \left(-\frac{\left(\Omega-w_{0}\right)^{2}}{4 \Gamma}\left[1-\frac{\Gamma(z)}{\Gamma}\right]\right) \cdot \exp \left[j\left(1-\frac{\Gamma(z)}{\Gamma}\right) \Omega+\frac{\Gamma(z)}{\Gamma} w_{0}\right]\left(t+\frac{z}{V_{g}(\Omega)}\right) .
\end{aligned}
$$

This wavelet is characterized by a Gaussian envelope. This decomposition is valid only for the values of $\Delta w$ much larger than $\delta w(\Delta w \gg \delta w)$.

The delay of group of the wavelet $\left[t+\frac{z}{V_{g}(\Omega)}\right]$ is characterized by a Gaussian envelope which is the temporal
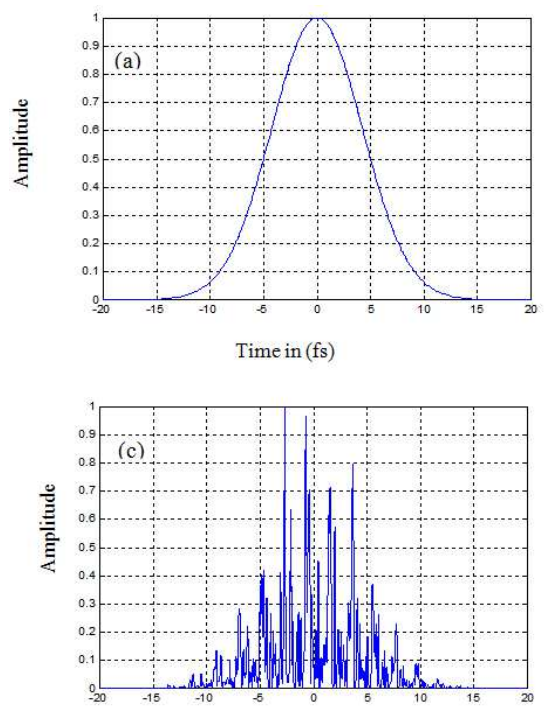

Time in (fs)

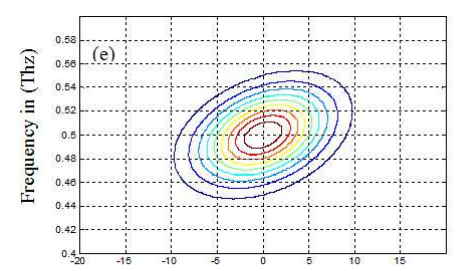

Time in (fs)

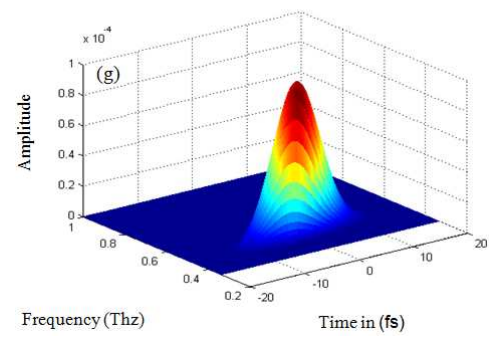

width.

The delay of group of the wavelet is inversely proportional to the velocity of group its envelope propagates without deformation (Khelladi, 2008).

\subsection{Simulations}

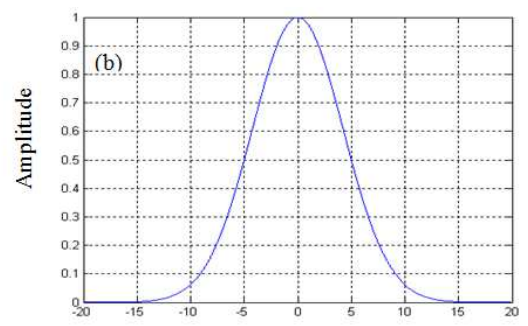

Time in $(\mathrm{fs})$

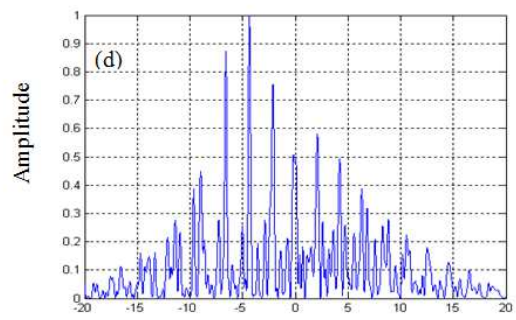

Time in (fs)

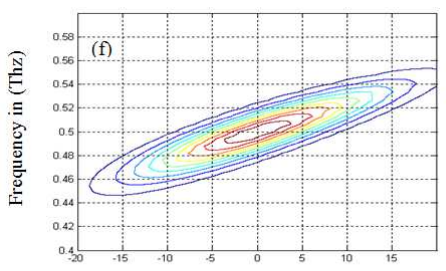

Time in $(\mathrm{fs})$

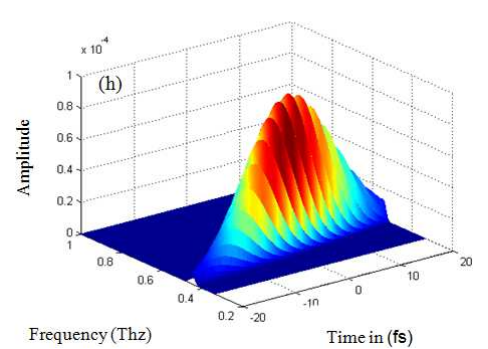

Fig 21. (a) Initial pulse, (c) pulse after propagation of the $20 \mathrm{~cm}$ in litharge medium, (e) Contour of the wavelet, ( $g$ ) the wavelet Representation, (b) Initial pulse, (d) pulse after propagation of the $20 \mathrm{~cm}$ in the silica medium, (f) Contour of the wavelet, (h) the wavelet Representation (Khelladi et al, 2008). 
Parameters of the simulations

Pulse initial: $\Delta \tau_{0}=10 \mathrm{fs}$

Wavelength : $\lambda=800 \mathrm{~nm}$

Pulse of the wavelet: $\Delta \tau_{\text {wavelet }}=1000 \mathrm{fs}$

Longer of the medium: $z=20 \mathrm{~cm}$

To describe the propagation of the pulse, we only consider the propagation of the maximum of each wavelet in a three dimensional representation:

\section{Conclusion}

In this article we have described powerful methods for crafting femtosecond optical pulses with precisely controllable temporal envelopes. For optical control the generation of light fields in a very flexible manner is desired such that all the different open-loop or closed-loop schemes can be implemented. In the case of femtosecond laser pulses this means that one should be able to manipulate the phase, amplitude, and possibly also the polarization-state properties with a large number of degrees of freedom. Ultrashort laser pulses can be described in the time domain and in the frequency domain which are connected via Fourier transforms, thus shaping of an ultrashort laser pulse can in principle be achieved either in the frequency or time domain.

Both phase and amplitude can be controlled, and great precision together with high complexity is possible. We began with a detailed discussion of generation of the ultrashort laser pulse and shaping methods which were largely developed by this author and his coworkers and which are now becoming widely adopted. We also reviewed a number of other pulse characterizations techniques, involving as FROG, SPIDER, VAMPIRE.

Finally, we have demonstrated here the possible decomposition of an ultrashort pulse into an infinite number of longer Fourier transform limited wavelets which propagate without any deformation through a dispersive medium. After propagation through the medium, the pulse may be visualized in a three dimensional representation by the locus of the wavelet maxima. This representation permits the evaluation of the broadening suffered by the pulse. For a transparent medium, the propagation of the $\Omega$ wavelet is described by the convolution of the incident $\Omega$ wavelet with a $\theta(\Omega)$ distribution centered at the group delay relative to $\Omega$.

The application to absorbing media is relatively straightforward and will be presented in a further publication, as well as a generalization to nonlinear media. The timefrequency representation is peculiarly suitable to the latter case for which the refractive index is phenomenological time dependent. Although this technique represents a vast improvement in our ability to describe such pulses, they require additional effort, both in the apparatus and in the extraction of the pulse intensity and phase from the experimental trace.

\section{Acknowledgments}

This work has been financially supported by the Electronics, and Telecommunications laboratory of Tlemcen University, Algeria.

\section{References}

[1] Khelladi.M, Seddiki.O, and Bendimerad.T, Time-Frequency Decomposition of an Ultrashort Pulse:Wavelet Decomposition, Radioengineering ISSN, April (2008) Vol.17, pp.1210-2512, N1

[2] Florenţa A.C, Dynamics of Ultra-short Laser Pulse Interaction with Solids at the Origin of Nanoscale Surface Modification" geboren am 16.11.1971 in Berca, Rumänien Cottbus (2006)

[3] Chritov, I.P., Propagation of femtosecond light pulses, Opt. Commun. 53, 364- 366

[4] Ziolokowski,R.W, Judkins, J.B. (1992)., Propagation characteristics of ultrawide bandwidth pulsed Gaussian beams, J.Opt. Soc. Am. A 11, 2021-2030, (1985).

[5] Sheppard, C.R, Xianosong, G, Free-space propagation of femtosecond ligthpulses, Opt. Commun. 133, 1-6. (1997)

[6] Agrawal, G.P, Spectrum-induced changes in diffraction of pulsed beams, Opt. Commun. 157, 52-56. (1998).

[7] Kaplan, A.E., Diffraction-induced transformation of near cycle and subcycle pulses, J. Opt. Soc. Am. B 15, 951-956. (1998)

[8] Agrawal, G.P, Far-Field diffraction of pulsed optical beams in dispersive media, Opt. Commun. 167, 15-22. (1999).

[9] Porras,M.A, Propagation of single-cycle pulse light beams in dispersive media, Phys. Rev. A 60, 5069-5073. (1998).

[10] Ichikawa, H, Analysis of femtosecond-order optical pulses diffracted by periodic structure, J. Opt. Soc. Am. A 16, 299304. (1999).

[11] Piestun, R., Miller, D.B., Spatiotemporal control of ultrashort optical pulses by refractive-diffractive-dispersive structured optical elements, Opt. let. 26, 1373-1375. (2001).

[12] Kempe, M., Stamm, U., Wilhelmi, B., and Rudolph, W., Spatial and temporal transformation of femtosecond laser pulses by lenses systems, J. Opt. Soc. 38, 1058-1064. (1999).

[13] Matei,G.O, Gili, M.A., Spherical aberration in spatial and temporal transformation of femtosecond laser pulses by lenses and lens systems, J. Opt. Soc. Am. B9, 1158-1165. (1992).

[14] Fuchss, U., Zeintner, U.D., and Tunnermenn (A.), Ultrashort pulse propagation in complex optical systems, Opt. Express 13, 3852-3861. (2005).

[15] Leonid.S., Ferrari.A, and Bertolotti.M, Diffraction of a time Gaussian-shaped pulsed plane wave from a slit, Pure. Appl. Opt5, 349-353. (1996).

[16] Hwang.H.E, Yang,G.H., Far-Field diffraction characteristics of a time variant Gaussian pulsed beam propagation from a circular aperture, Opt. Eng. 41, 2719-2727. (2002). 
[17] Liu,Z, Lu,B., Spectral shifts and spectral switches in diffraction of ultrashort pulsed beams passing through a circular aperture, Optik 115, 447-454, (2004).

[18] Abdolah.M.K, "Manipulation and characterization of femtosecond laser pulses for cluster spectroscopy" vorgelegt von aus Teheran (Iran) July (2007).

[19] Ruiz-de-la-Cruz.A and R. Rangel-Rojo, Multi-pass confocal ultra-short pulse amplifier Revista Mexicana de Fisica 51 (5) 488-493, M'exico (2005)

[20] Joanna.M, "Seutp of very advanced for phase and amplitude reconstruction of electric field (VAMPIRE)" Master Thesis submitted to the Faculty for the Natural Sciences and for Mathematics of the Rostock University Germany (2007).

[21] Holzwarth, "Measuring the Frequency of Light using Femtosecond Laser Pulses" aus Stuttgart den 21. December (2000).

[22] Hofmann.A, Bestimmung des elektrischen Feldes ultrakurzer Laserpulse mit SPIDER, Teill: Theorie, Projektpraktikumsbericht, Physikalisches Institut, EP1, Universität Würzburg (2005).

[23] Rulliere, C, Femtosecond laser pulses: principles and Experiments, Springer, (1998).

[24] Jong Kook K. Investigation of high nonlinearity glass fibers for potential applications in ultrafast nonlinear fiber devices, Dissertation submitted to the Faculty of the Virginia (2005).

[25] Meyer,Y, Jaffort, S., Riol,O, wavelet analysis, edition Française de scientifique American (1987)

[26] Khelladi,M. Ultra-short lasers pulses Characterizations: Wavelet decomposition, , University of Tlemcen. Algeria, 2005.

[27] Moulton.P.f, Quant. Electron. Conf., Munich, Germany, June (1982).
[28] Greg.T, Andy. R Margaret.M, Measurement of 10 fs Laser Pulses. 1077-260X, IEEE (1996).

[29] Marco.A, Krumbugel.Kenneh.W,and Trebino. R, frequency resolved optical gating measurements of ultra short pulses using surface third harmonic generation, Optics Letters, Vol21, $\mathrm{N}^{\circ} 17$, September (1996).

[30] Chritov, I. P. Propagation of femtosecond light pulses. Opt. Commun., 1985, vol. 53, no. 6, pp. 364- 366 .

[31] Agrawal, G. P. Spectrum-induced changes in diffraction of pulsed beams. Opt. Commun., 1998, vol. 157, pp. 52-56.

[32] Kaplan, A. E. Diffraction-induced transformation of near cycle and subcycle pulses. J. Opt. Soc. Am. B, 1998, vol. 15, pp. 951-956.

[33] Agrawal, G. P. Far-field diffraction of pulsed optical beams in dispersive media. Opt. Commun., 1999, vol. 167, pp. 15-22.

[34] Porras, M.A. Propagation of single-cycle pulse light beams in dispersive media. Phys. Rev. A, 1999, vol. 60, no. 6, pp. 50695073.

[35] Ichikawa, H. Analysis of femtosecond-order optical pulses diffracted by periodic structure. J. Opt. Soc. Am. A, 1999, vol. 16, pp.299-304.

[36] Piestun, R., Miller, D. B. Spatiotemporal control of ultrashort optical pulses by refractive-diffractive-dispersive structured optical elements. Opt. Let., 2001, vol. 26, pp. 1373-1375.

[37] Ssheppard, C. J. R., GAN , X. Free-space propagation of femtosecond light pulses. Opt. Commun., 1997, vol. 133, pp. $1-6$.

[38] Kempe, M., Stamm, U., Wilhelmi, B., Rudolph, W. Spatial and temporal transformation of femtosecond laser pulses by lenses systems. J. Opt. Soc., 1999, vol. 38, pp. 1058-1064. 